\title{
4-valent plane graphs with 2-, 3- and 4-gonal faces
}

\author{
Michel DEZA \\ CNRS/ENS, Paris and Institute of Statistical Mathematics, Tokyo, \\ Mathieu DUTOUR \\ ENS, Paris and Hebrew University, Jerusalem, * \\ Mikhail SHTOGRIN ${ }^{\dagger}$ \\ Steklov Mathematical Institute, Moscow, Russia.
}

October 29, 2018

\begin{abstract}
Call $i$-hedrite any 4 -valent $n$-vertex plane graph, whose faces are 2 -, 3 - and 4 -gons only and $p_{2}+p_{3}=i$. The edges of an $i$-hedrite, as of any Eulerian plane graph, are partitioned by its central circuits, i.e. those, which are obtained by starting with an edge and continuing at each vertex by the edge opposite the entering one. So, any $i$-hedrite is a projection of an alternating link, whose components correspond to its central circuits.

Call an $i$-hedrite irreducible, if it has no rail-road, i.e. a circuit of 4-gonal faces, in which every 4-gon is adjacent to two of its neighbors on opposite edges.

We present the list of all $i$-hedrites with at most 15 vertices. Examples of other results:

(i) All $i$-hedrites, which are not 3-connected, are identified.

(ii) Any irreducible $i$-hedrite has at most $i-2$ central circuits.

(iii) All $i$-hedrites without self-intersecting central circuits are listed.

(iv) All symmetry group of $i$-hedrites are listed.

Mathematics Subject Classification. Primary 52B05, 52B10; Secondary 05C30,

Key words. Plane graphs, Eulerian graphs, alternating links, point groups.
\end{abstract} $05 \mathrm{C} 10$

* Research of the second author was financed by EC's IHRP Programme, within the Research Training Network "Algebraic Combinatorics in Europe," grant HPRN-CT-2001-00272.

${ }^{\dagger}$ Third author acknowledges financial support of the Russian Foundation of Fundamental Research (grant 02-01-00803) and the Russian Foundation for Scientific Schools (grant 00-15-96011) 


\section{Introduction}

See [Grün67] for terms used here for plane graphs. It is well-known that the p-vector of any 4 -valent plane graph satisfies to $2 p_{2}+p_{3}=8+\sum_{i>5}(i-4) p_{i}$. Some examples of applications of plane 4-valent graphs are projections of links, rectilinear embedding in VLSI and Gauss crossing problem for plane graphs (see, for example, Liu98]).

Call an $i$-hedrite any plane 2-connected 4-valent graph, such that the number $p_{j}$ of its $j$-gonal faces is zero for any $j$, different from 2,3 and 4 , and such that $p_{2}=8-i$. So, an $n$-vertex $i$-hedrite has $\left(p_{2}, p_{3}, p_{4}\right)=(8-i, 2 i-8, n+2-i)$. Clearly, $\left(i ; p_{2}, p_{3}\right)=(8 ; 0,8),(7 ; 1,6),(6 ; 2,4),(5 ; 3,2)$ and $(4 ; 4,0)$ are all possibilities.

An 8-hedrite is called octahedrite in DeSt02; in fact, this paper is a follow-up of DeSt02. In a way, this paper continues the program of Kirkman (Kir85] p. 282) of classification of projections of alternating links.

See on the Table below short presentation of $i$-hedrites and their symmetry groups. In the last column we indicate Goldberg-Coxeter operation $G C_{k, l}\left(G_{0}\right)$ starting from $i$-hedrite $G_{0}$ with smallest number of vertices (see Gold37, [Cox71] and [DD03]).

For 3-connected plane graphs without 2-gons, the following Theorem of Mani is valid: the symmetry group of the graph can be realized as point group of a convex polyhedron having this graph as skeleton. But in the presence of 2-gonal faces, one cannot speak of convex polyhedra but we expect that the Mani result still holds for this more general case.

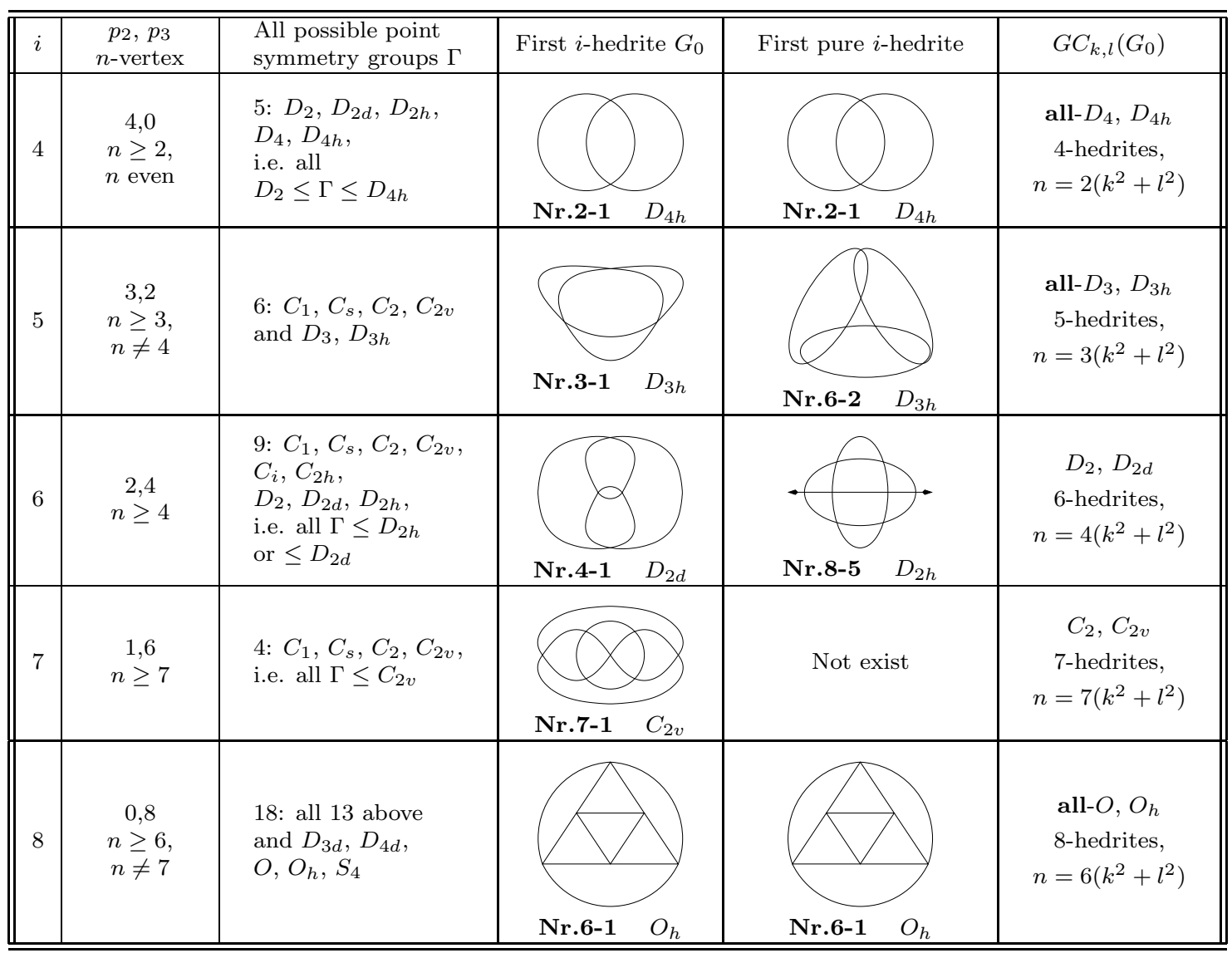




\section{Central circuits partition}

In this Section, we consider a connected plane graph $G$ with all vertices of even degree, i.e. an Eulerian graph. Call a circuit in $G$ central if it is obtained by starting with an edge and continuing at each vertex by the edge opposite the entering one; such circuit is called also traverse (GaKe94]), straight ahead ([Harb97]), [PTZ96]), straight Eulerian (Chapter 17 of GoRo01]), cut-through ([Jeo95]), intersecting, etc. Clearly, the edge-set of $G$ is partitioned by all its central circuits.

Such CC-partition can be considered (see, for example, [Harb97]) for any drawing on the plane of any Eulerian (in general, not planar) graph, so that edges are mapped into simple curves with at most one crossing point.

Denote by $C C(G)=\left(\ldots, a_{i}^{\alpha_{i}}, \ldots ; \ldots, b_{j}^{\beta_{j}}, \ldots\right)$ its $C C$-vector, where $\ldots, a_{i}, \ldots$ and $\ldots, b_{j}, \ldots$ are increasing sequences of lengths of all its central circuits, simple ones and self-intersecting, respectively, and $\alpha_{i}, \beta_{j}$ are their respective multiplicities. Clearly, $\sum_{i} a_{i} \alpha_{i}+\sum_{j} b_{j} \beta_{j}=2 n$, where $n$ is the number of vertices of $G$.

For a central circuit $C$, denote by $\operatorname{Int}(C):=\left(c_{0} ; \ldots, c_{k}^{\gamma_{k}}, \ldots\right)$, the intersection vector of $C$, where $c_{0}$ is the number of self-intersections of the circuit $C$ and $\ldots, c_{k}, \ldots$ is decreasing sequence of sizes of its intersection with other central circuits, while the numbers $\gamma_{k}$ are respective multiplicities.

Two central circuits intersect in an even number number of vertices. The length of a central circuit is twice the number of its points of self-intersection plus the sum of its intersections with other circuits, so the length of a central circuit is even.

We will say that an $i$-hedrite is pure if any of its central circuits simple, i.e. has no self-intersections. Easy to check that any pure $i$-hedrite has an even number $n$ of vertices. In fact, any vertex in this case belong to the intersection of exactly two central circuits.

Call an Eulerian graph $G$ balanced, if all its central circuits of same length have the same intersection vector. Any 8 -hedrite with $n \leq 21$ is balanced, but there is unbalanced 22-vertex 8-hedrite, which is 8-hedrite 14-7 of Table 2 inflated along a central circuit of length 8 . We do not find unbalanced 5-hedrite or 7-hedrite for $n \leq 15$. The first unbalanced 6-hedrite is 12-12. Any 4-hedrite is balanced (Theorem [5).

For a plane graph $G$, denote by $G^{*}$ its plane dual and by $\operatorname{Med}(G)$ its medial graph. The vertices of $\operatorname{Med}(G)$ are the edges of $G$, two of them being adjacent if the corresponding edges share a vertex and belong to the same face of the embedding of $G$ in the plane. So, $\operatorname{Med}(G)=\operatorname{Med}\left(G^{*}\right)$.

Clearly, $\operatorname{Med}(G)$ is a 4 -valent plane graph and, for any $i$-hedrite $G, \operatorname{Med}(G)$ is an $i$-hedrite with twice the number of vertices of $G$ and all 2 -, 3-gonal faces being isolated. The medial of smallest 8-hedrite $\mathbf{6 - 1}, \mathbf{7}$-hedrite $\mathbf{7 - 1}, 6$-hedrite $\mathbf{4 - 1}, \mathbf{5}$-hedrite 3-1, 4-hedrite 3-1 are, respectively, 8-hedrite $\mathbf{1 2 - 4}$, 7-hedrite $\mathbf{1 4 - 9}$, 6-hedrite $\mathbf{8 - 3}$, 5-hedrite 6-2, 4-hedrite 4-1. The operation of taking the medial is a particular case of the Goldberg-Coxeter construction for the parameters $(k, l)=(1,1)$ (Gold37, Cox71], DD03]). 


\section{Intersection of central circuits}

The following Theorem is a local version (for "parts" of the sphere) of the Euler formula $2 p_{2}+p_{3}=8+\sum_{i \geq 5}(i-4) p_{i}$ for $p$-vector of any 4 -valent plane 3 -connected graph $P$.

For any 4-valent planar graph $P$, a patch $A$ is a region of $P$ bounded by $q$ arcs (paths of edges) belonging to central circuits (different or coinciding), such that all $q$ arcs form together a circle. A patch can be seen as a $q$-gon; we admit also 0-gonal $A$, i.e. just the interior of a simple central circuit. Suppose that the patch $A$ is regular, i.e. the continuation of any of bounding arc (on the central circuit to which it belongs) lies outside of the patch. See below two examples of patch.
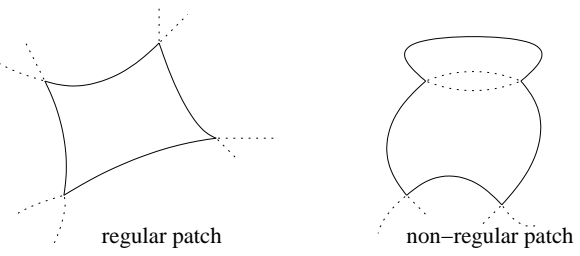

Let $p^{\prime}(A):=p_{1}^{\prime}, \ldots$ be the $p$-vector enumerating the faces of the patch $A$. The curvature of the patch $A$ is defined as $c(A)=\sum_{k \geq 1}(4-k) p_{k}^{\prime}$. So, the $k$-gon can be seen as, respectively, positively curved, flat, or negatively curved, if $k<4, k=4$, or $k>4$.

Proposition 1 (i) If $A$ be a regular patch, then $c(A)=4-q$, moreover:

(i.1) $c(A)=4$ if and only if $A$ is bounded by a simple central circuit.

(i.2) $c(A)=0$ if and only if $A$ is a rectangle formed by 4-gons put together.

(ii) Any patch $A$ is the union of regular patches $A_{1}, \ldots, A_{p}$; one has $c(A)=$ $\left(4-q_{1}\right)+\cdots+\left(4-q_{p}\right)$, where each patch $A_{i}$ is bounded by $q_{i}$ arcs.

(iii) If a graph $G$ is the union of patches $A_{1}, \ldots, A_{p}$, then $8=c\left(A_{1}\right)+\cdots+c\left(A_{p}\right)$.

Proof. (i) is a restatement in our terms of Theorem 1 of [DeSt02, (i1) and (i2) are easy consequences. The properties (ii) and (iii) follow from the definition of the curvature of a patch and from Euler formula.

Proposition 2 (i) Any 4-valent plane graph whose faces are $k$-gons with $k$ even has central circuits with no self-intersection vertices.

(ii) At least one central-circuit of a 7-hedrite self-intersects.

Proof. In fact, if a central circuit of a 4-hedrite self-intersects, then we have an 1-gonal regular patch. The equality of above Theorem becomes $\sum_{i}(4-i) p_{i}^{\prime}=3$, an impossibility since the left hand side is even.

Take a central circuit containing an edge of the unique 2-gon, then the sequence (possibly empty) of adjacent 4-gons will necessarily finish by a 3-gon, or this 2-gon; both cases yield a self-intersection.

Let us call graph of curvatures of an $i$-hedrite $G$, the graph (possibly, with loops and multiple edges) having as vertex-set all 2 -gons and 3 -gons of $G$. Two vertices 
(say, 2- or 3-gonal faces $F$ and $F^{\prime}$ of $G$ ) of this $i$-vertex graph are adjacent if there is a pseudo-road connecting them. A pseudo-road is sequence of 4 -gons, say, $F_{1}, \ldots, F_{l}$, such that putting $F_{0}=F$ and $F_{l+1}=F^{\prime}$, we have that any $F_{k}$ with $1 \leq k \leq l$ is adjacent to $F_{k-1}$ and $F_{k+1}$ on opposite edges (cf. the definition of a rail-road in next Section). Clearly, in the graph of curvatures, the vertices corresponding to 2- and 3 -gons, have degree 2 and 3, respectively.

Proposition 3 Let $C_{1}, C_{2}$ be any two central circuits of an $i$-hedrite. Then they are disjoint if and only if they are simple and there exist a ring of 4-gons separating them.

Proof. In fact, if both $C_{1}$ and $C_{2}$ are simple circuits, Theorem is evident: the curvature of the interior of a patch is 4 and so, two circuits are separated by 4-gons only. Suppose that $C_{1}$ is self-intersecting. Then it has at least three regular patches and each of them has curvature at most 3 . The circuit $C_{2}$, being disjoint with $C_{1}$, lies entirely inside one of those patches, say, $A$. So, all its 3 -gons and 2-gons, except, possibly, those from its exterior patch, lie in $A$. So, $c(A) \geq 5$, since the exterior patch of $C_{2}$ has curvature at most 3 . It contradicts to the fact that $A$ has curvature at most 3 .

Remark 1 Consider a 4-valent plane graph $G$ having only one central circuit; then, the set of faces of $G$ can be partitioned into two classes $\mathcal{C}_{1}, \mathcal{C}_{2}$ in chess manner. Every vertex $v$ is contained in two faces $F$ and $F^{\prime}$. Also, the unique central circuit can be given an orientation, which induces an orientation on the set of edges.

The vertex $v$ is incident to two edges of $F, e_{1}$ and $e_{2}$, and to two edges of $F^{\prime}, e_{1}^{\prime}$ and $e_{2}^{\prime}$. If $e_{1}, e_{2}$ have both arrow pointing to the vertex or both arrow pointing out of the vertex, then the same is true for $e_{1}^{\prime}$ and $e_{2}^{\prime}$, and then, we say that $v$ belongs to Class I. Class I and its complement, Class II, form a bipartition of the set of vertices of the knot; reversing orientation of the central circuit or interchanging $\mathcal{C}_{1}$ and $\mathcal{C}_{2}$ does not change the bipartition.

If the graph consists of $p, p \geq 2$, central circuits $C_{1}, \ldots, C_{p}$, then, one can put orientations on every central circuit and get a bipartition of the set of vertices. But in that case the bipartition will depend on the chosen orientations.

\section{Adding and removal central circuits}

The deleting of a central circuit $C$ in an $i$-hedrite $G$ consists of removal of all edges and vertices contained in $C$. It produces a 4 -valent plane graph $P^{\prime}$ having only $k$-gonal faces with $k \leq 4$. But since cases $k=0,1$ are possible, we do not always obtain an $i$-hedrite.

The cutting of an $i$-hedrite $G$ consists of adding another central circuit to it. The faces of the new $i^{\prime}$-hedrite $G^{\prime}$ with $8 \geq i^{\prime} \geq i$ comes from the cutting of faces of $G$. This operation is only partially defined, since arbitrary cutting can produce $k$-gons with $k>4$. The cutting of a 4 -gon in several 4 -gons (two, if the face is traversed only once) is possible only if the 4-gon is traversed on opposite edges. 
This corresponds to the notion of shore-zone in DeSt02]. A cutting changes CCpartition of an $i$-hedrite only in the following way: new central circuit $C$ is added and all others central circuits remain unchanged, except that the length of each of them increases by one for any intersection with $C$.

Call a rail-road a circuit of 4-gons, possibly self-intersecting, in which every 4gon is adjacent to two of its neighbors on opposite edges. A rail-road is bounded by two "parallel" central circuits. The deleting of one of those central circuits (in other words, collapsing rail-road into one central circuit) is called reduction. The cutting produces a rail-road if and only if it is an inflation along a central circuit $C$, i.e. replacing it by (thin enough) rail-road. A t-inflation along a central circuit $C$ is replacing this central circuit by $t-1$ parallel (thin enough) rail-roads. A $t$ inflation of an $i$-hedrite is new $i$-hedrite obtained from original one by simultaneous $t$-inflation along all of its central circuits. A $t$-inflation of $G$ is $G$ if $t=1$, and it is just inflation of $G$ if $t=2$.

An $i$-hedrite is called irreducible if it contains no rail-road. It is called maximal irreducible if it cannot be obtained from another $i^{\prime}$-hedrite by a cutting.

Remark 2 Let $C$ be a central circuit of $G$ with $C C(G)=\left(\ldots, a_{i}^{\alpha_{i}}, \ldots ; \ldots, b_{j}^{\beta_{j}}, \ldots\right)$, and let $\operatorname{Int}(C)=\left(c_{0} ; c_{1}^{\gamma_{1}}, \ldots, c_{r}^{\gamma_{r}}\right)$. The t-inflation of $G$ denoted by $G^{t}$ has $C C\left(G^{t}\right)=$ $\left(\ldots, t a_{i}^{t \alpha_{i}}, \ldots ; \ldots, t b_{j}^{t \beta_{j}}, \ldots\right)$; if $C^{\prime}$ is one of $t$ parallel copies of $C$, then $\operatorname{Int}\left(C^{\prime}\right)=$ $\left(c_{0} ; c_{1}^{t \gamma_{1}}, \ldots, c_{r}^{t \gamma_{r}},\left(2 c_{0}\right)^{t-1}\right)$.

\section{Connectivity of $i$-hedrites}

For any integer $m \geq 2$ denote:

by $I_{6,2 m}$ the $2 m$-vertex 6 -hedrite, such that each 2 -gon is adjacent to two 3 -gons;

by $I_{5,2 m+1}$ the $(2 m+1)$-vertex 5 -hedrite, such that two 2 -gons share a vertex and remaining 2 -gon is adjacent to two 3 -gons;

by $I_{4,2 m+2}$ the $(2 m+2)$-vertex 4 -hedrite, such that four 2-gons are organized into two pairs sharing a vertex;

by $J_{4,2 m}$ the $m$-inflation of only one central circuit of 4 -hedrite $\mathbf{2 - 1}$; they are projections of composite alternating links $2_{1}^{2} \# 2_{1}^{2} \# 2_{1}^{2} \ldots \# 2_{1}^{2}$ ( $m$ times), which we denote by $m \times 2_{1}^{2}$.

See in Table 1 the first occurrences (for $2 \leq m \leq 5$ ) of those graphs, followed by their symmetry groups and CC-vectors.

Lemma 1 Any i-hedrite is 2-connected.

Proof. Let $G$ be an $i$-hedrite and assume that there is one vertex $v$, such that $G-\{v\}$ is disconnected in two components $C_{1}$ and $C_{2}$. Then two edges from $v$ will connect to a vertex $w$ of $C_{1}$ and two edges from $v$ will connect to a vertex $w^{\prime}$ of $C_{2}$, because, otherwise, the exterior face is $m$-gonal with $m>4$. See below the corresponding drawing. 

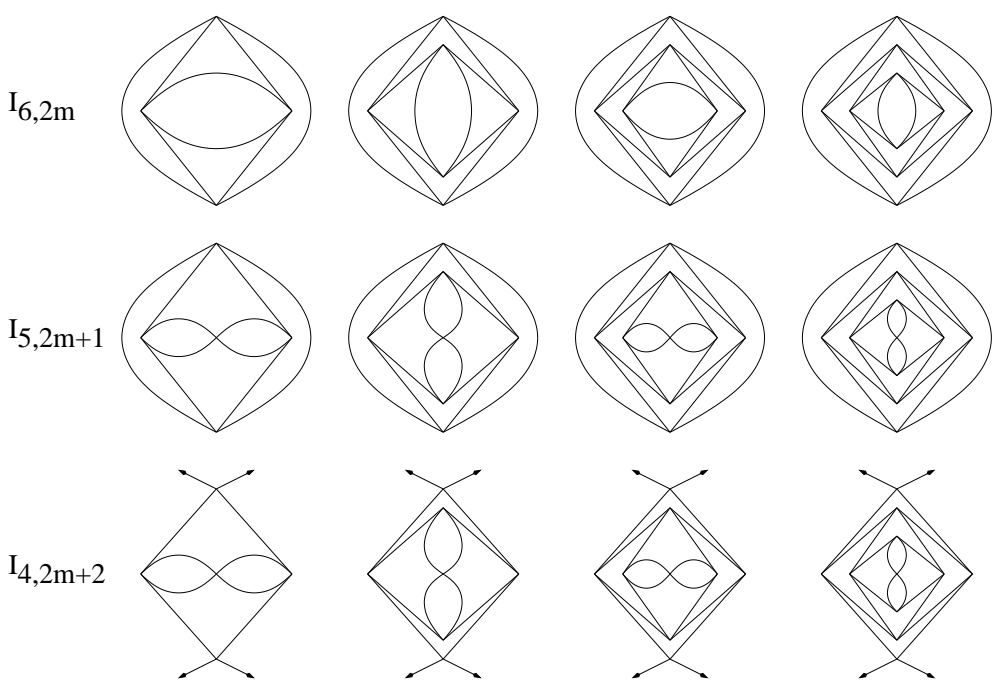

$\mathrm{J}_{4,2 \mathrm{~m}}$
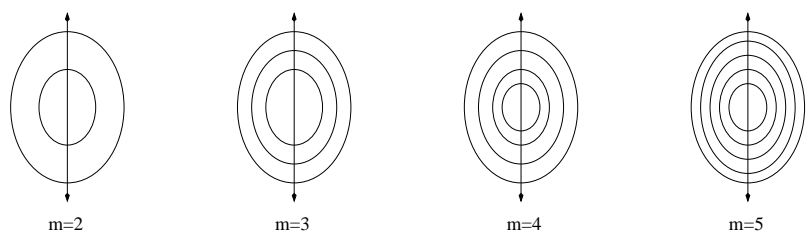

\begin{tabular}{||c|c|c||}
\hline \hline$i$-hedrite & Group & CC-vector \\
\hline \hline$I_{6,2 m}, m$ even & $D_{2 d}$ & $4 m$ \\
\hline$I_{6,2 m}, m$ odd & $D_{2 h}$ & $(2 m)^{2}$ \\
\hline$I_{5,2 m+1}$ & $C_{2 v}$ & $4 m+2$ \\
\hline$I_{4,2 m+2}, m$ even & $D_{2 d}$ & $(2 m+2)^{2}$ \\
\hline$I_{4,2 m+2}, m$ odd & $D_{2 h}$ & $(2 m+2)^{2}$ \\
\hline$J_{4,2 m}$ & $D_{2 h}$ & $2^{m}, 2 m$ \\
\hline \hline
\end{tabular}

Table 1: All $i$-hedrites, which are not 3-connected 


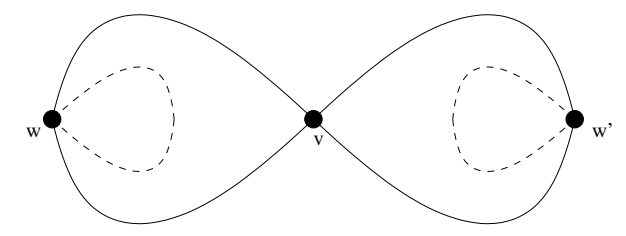

But the vertex $w$ will disconnect the graph and so, iterating the construction, we obtain an infinite sequence $v_{1}, \ldots, v_{n}$ of vertices that disconnects $G$. This is impossible since $G$ is finite.

Any $i$-hedrite (moreover, any Eulerian graph) has at least one Eulerian circuit of edges; so, there is no cut-edge. But a cut-vertex appears already for some Eulerian (3-vertex) and 4-valent (4-vertex) plane graphs.

Theorem 1 Any i-hedrite, which is not 3 -connected, is one of $I_{6,2 m}, I_{5,2 m+1}, I_{4,2 m+2}$, $J_{4,2 m}$ for some $m \geq 2$.

Proof. Let $G$ be an $i$-hedrite and assume that it is not 3-connected. Then there are two vertices, say, $v$ and $v^{\prime}$, such that $G-\left\{v, v^{\prime}\right\}$ is disconnected in two components, say, $C_{1}$ and $C_{2}$. Amongst the 4 edges from $v$ (respectively $v^{\prime}$ ), the edges $\left\{e_{1}, \ldots, e_{s}\right\}$ (respectively $\left\{e_{1}^{\prime}, \ldots, e_{s^{\prime}}^{\prime}\right\}$ ) go to $C_{1}$. Two numbers $s$ and $s^{\prime}$ can takes values 1,2 or 3 ; we will consider all possible cases.

Assume that $s=1$ and $s^{\prime}=1$, then the edges $e$ and $e^{\prime}$ must be distinct, since, otherwise, $C_{1}$ is the empty graph. Moreover, $e$ and $e^{\prime}$ have no common vertices, since, otherwise, $G$ would not be 2 -connected. So, $v$ and $v^{\prime}$ are connected by $e$ and $e^{\prime}$ to a vertex $w$ and $w^{\prime}$, respectively. Since face-size is at most 4 , the vertices $v$ and $v^{\prime}$, (respectively, $w$ and $w^{\prime}$ ) are linked by two edges (see Figure 1). Two points $w$ and $w^{\prime}$ can either be connected by two edges and we are done, or disconnect the graph. In the latter case, we can iterate the construction. Since the graph is finite, the construction eventually finish and we get a graph $J_{4,2 m}$. If $s=1$ and $s^{\prime}=3$, then by a similar reasoning, one gets again a graph $J_{4,2 m}$.

Assume that $s=2$ and $s^{\prime}=2$. One has $\left\{e_{1}, e_{2}\right\} \cap\left\{e_{1}^{\prime}, e_{2}^{\prime}\right\}=\emptyset$, since, otherwise, one can attribute an edge to $C_{2}$ and get the case $s=1$ and $s^{\prime}=1$. So, one has, say, $e_{1} \cap e_{1}^{\prime}=\left\{w_{1}\right\}$ and $e_{2} \cap e_{2}^{\prime}=\left\{w_{2}\right\}$, and the following two possibilities (see Figure 11): either $w_{1}=w_{2}$ (this corresponds to $\left\{e_{1}, e_{2}\right\}$ and $\left\{e_{1}^{\prime}, e_{2}^{\prime}\right\}$ being the edges of two 2 -gons) and we are done, or $w_{1} \neq w_{2}$. Assume now that $w_{1} \neq w_{2}$; two points $w_{1}$ and $w_{2}$ can either be connected by two edges and we are done, or disconnect the graph. In the latter case, we can iterate the construction. Since the graph is finite, the construction eventually finish. If we do the same construction on the other side, then we get a similar structure and the graph is of the form $I_{4,2 m+2}, I_{5,2 m+1}$ or $I_{6,2 m}$ with $m \geq 2$.

Assume now that $s=2$ and $s^{\prime}=1$. The edges $e_{1}, e_{2}$ and $e_{1}^{\prime}$ are all distinct, since, otherwise, the vertex $v$ disconnects the graph. So, $v^{\prime}$ is connected by $e_{1}^{\prime}$ to a vertex $w^{\prime}$. Now, either $v^{\prime}$ or $w^{\prime}$ is connected to $v$, since, otherwise, we would have a 5-gonal face. If $w^{\prime}$ is connected to $v$, then the pair $\left\{w^{\prime}, v\right\}$ disconnects the graph. This construction is infinite (see Figure 11); so, we get a contradiction. 


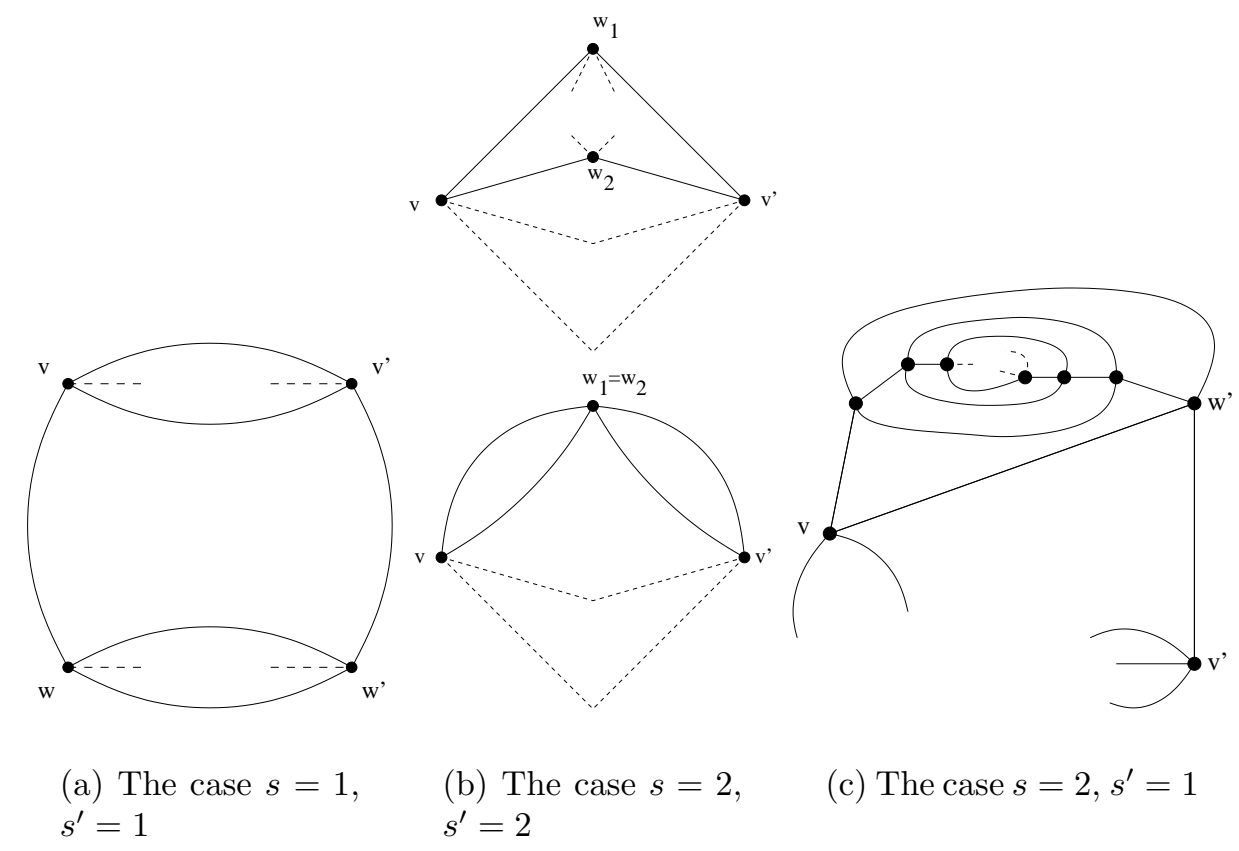

Figure 1: The three cases of Theorem 1

Theorem 2 (i) If $G$ is an i-hedrite with two adjacent 2-gons, then this is a 4-hedrite 2-1 or a $J_{4,2 m}$ with $m \geq 2$.

(ii) If $G$ is an $i$-hedrite with two 2-gons sharing a vertex, then it is either 4-hedrite 4-1, or an $I_{4,2 m+2}$, or 5-hedrite $3-1$, or an $I_{5,2 m+1}$ with $m \geq 2$.

Proof. The proof for (i), (ii) is similar to the cases $\left(s, s^{\prime}\right)=(1,1),(2,2)$ of Theorem 11.

Theorem 3 (i) an n-vertex 4-hedrites exists if and only if $n \geq 2$, even.

(ii) an n-vertex 5-hedrites exists if and only if $n \geq 3, n \neq 4$.

(iii) an n-vertex 6 -hedrites exists if and only if $n \geq 4$.

(iv) an n-vertex 7 -hedrites exists if and only if $n \geq 7$.

(v) an $n$-vertex 8-hedrites exists if and only if $n \geq 6, n \neq 7$.

Proof. The case (v) is proven in Grün67, page 282. The case (i) is trivial; take, for example, the serie $J_{4,2 m}$.

The series $I_{6,2 m}, I_{5,2 m+1}$ for any $m \geq 2$, give 6 -hedrites and 5-hedrites with even and, respectively, odd number of vertices.

For 5-, 6- and 7-hedrites, we get by $(t-1)$-inflation along a central circuit of length 4 in corresponding $i$-hedrite $\mathbf{6 - 1}, \mathbf{5 - 1}$ and $\mathbf{7 - 1}$, series with $4 t+2,4 t+1$ and $4 t+3$ vertices. By $(t-2)$-inflation along such central circuit in 7 -hedrite $\mathbf{8 - 1}$, we get a serie of 7 -hedrites with $4 t$ vertices. By $(t-1)$-inflation along central circuit in 6 -hedrite 11-4, we get serie of 6 -hedrites with $8 t+3$ vertices. By $t$-inflation along central circuit of length 8 in 6 -hedrite 15-10, we get serie of 6 -hedrites with $8 t+7$ vertices. 
Inscribing consecutively 4-gons in the 4-gon, which is adjacent only to 3-gons, in 7-hedrite $\mathbf{9 - 1}$ and $\mathbf{1 0 - 2}$, we get series of 7-hedrites for the remaining cases of $4 t+1$ and $4 t+2$ vertices.

In case of 5 -hedrites, it remains to prove existence for the case $n=4 t>1$. We obtain existence in the sub-cases $n=m \times 4^{b}$, where $m \geq 3$ and not divisible by 4 , $n=8 \times 4^{b}$, and $n=16 \times 4^{b}$, respectively:

by $b$-inflation of some $m$-vertex 5 -hedrite; we showed their existence,

by $b$-inflation of 5 -hedrites $\mathbf{8 - 1}$,

by $b$-inflation of any 16-vertex 5 -hedrite (for example, one coming from 10-2 by inflation along central circuit of length 6 ).

Our computation (see the last Section) present all $i$-hedrites with at most 15 vertices.

\section{$6 \quad$ Irreducible $i$-hedrites}

Theorem 4 Any irreducible $i$-hedrite has at most $i-2$ central circuits and equality is attained for each $i, 4 \leq i \leq 8$.

Proof. For $i=8$, the Theorem is proved in DeSt02. We will show, using suitable cutting, that this result implies the Theorem for $i<8$.

Let us start with the simplest case of 7 -hedrites. Consider a simple circuit $S$ in its curvature graph, which contains the vertex corresponding to unique 2-gon. Remind that the vertices in the curvature graph correspond to 2- or 3-gons, while edges correspond to pseudo-roads. So, the simple circuit $S$ corresponds to the circuit of faces of $G$, containing our 2-gon, some 4-gons and, possibly, some of six 3-gons; see the picture below.

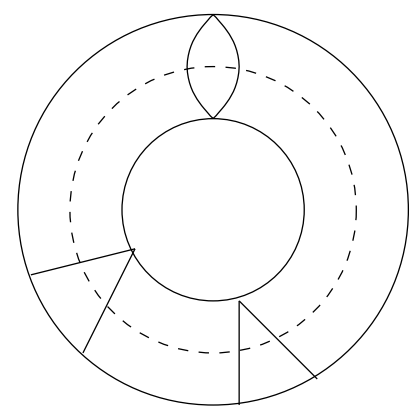

Suppose that the 7-hedrite $G$ is irreducible and has $k$ central circuits. By adding the central circuit $C$, which is shown by dotted lines on picture above, we produce an 8-hedrite (since, the 2-gon is cut by $C$ in two 3 -gons), which is still irreducible and has $k+1$ central circuits. So, $k+1 \leq 6$, by Theorem 3 of DeSt02].

For remaining cases of $i$-hedrites with $i=4,5,6$, the proof is similar. In each case, we consider all possible distribution of 2-gons by simple circuits in the graph of curvatures and, for each such circuit, we add suitable number of new central circuits.

All possibilities are presented on Figure 2 two for $i=6$, three for $i=5$ and two for $i=4$. In the last case, there are no 3 -gons and so, simple circuits in the curvature 


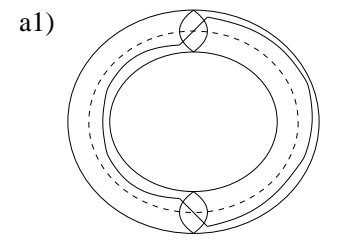

a2)

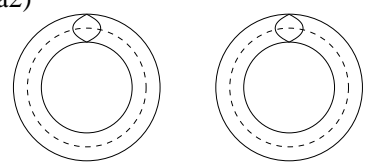

(a) The two cases for 6-hedrites

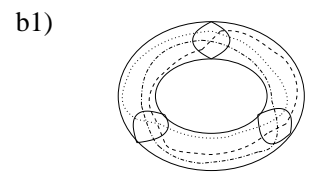

b2)

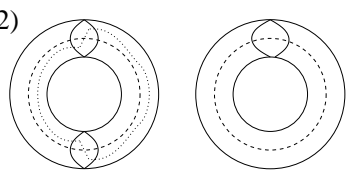

b3)

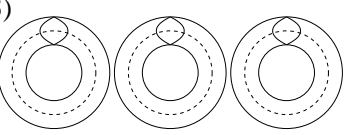

(b) The three cases for 5-hedrites

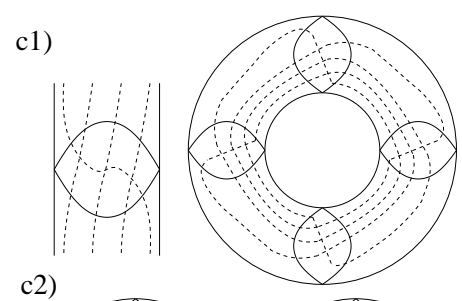

c2)

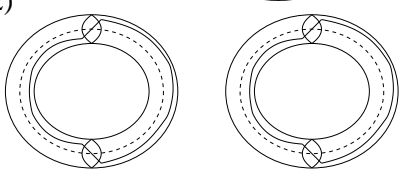

(c) The two cases for 4hedrites

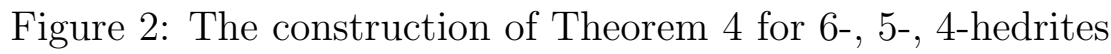

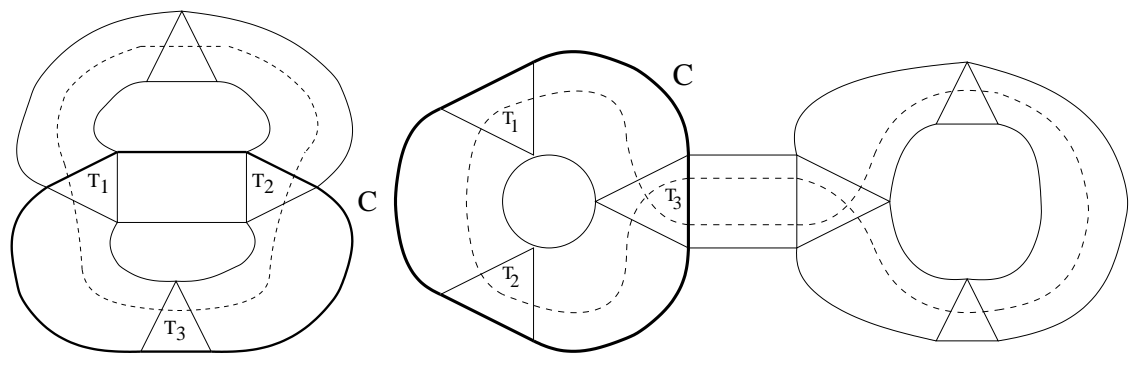

(a) The first case

(b) The second case

Figure 3: The two cases of cutting of irreducible 8-hedrite

graph contain only even number of 2-gons by local Euler formula of Theorem 1. Note that the case of 4 -hedrites is obvious by Theorem 5 of [DeSt02].

Lemma 2 Let $G$ be an irreducible 8-hedrite and $C$ a central circuit, which is incident to three 3-gons on one side. Then one can add another central circuit to $G$, so that the resulting graph is still irreducible.

Proof. From every one of the three 3 -gons, say, $T_{1}, T_{2}, T_{3}$, one can define two pseudo-roads from the sides of the 3 -gon, which do not belong to the central circuit. Each such pseudo-road defines an edge, say, $e_{1}, e_{2}, e_{3}$ in the graph of curvatures and so, a triangle in that graph. Then, either two triangles $T_{i}$ and $T_{j}$ are linked by a path, which does not involve the edges $e_{k}$, or they are not linked by such a patch. In both cases we can cut the 8-hedrite according to Figure 3 and obtain another 8-hedrite, which is still irreducible.

See below example of an irreducible 7 -hedrite (its CC-vector is $\left(10^{2}, 12^{2} ; 20\right)$, its symmetry group is $C_{2 v}$ ) with the maximum number of central circuits. 


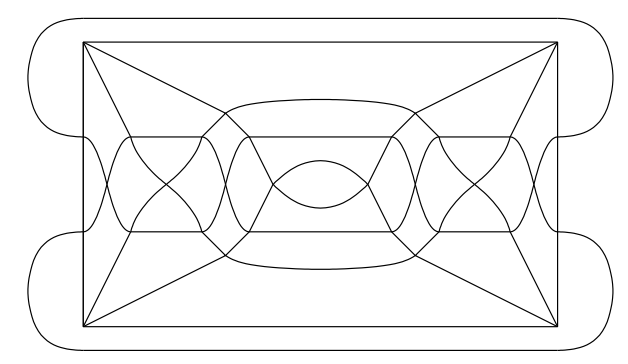

For all other $i$, there is an example of irreducible $i$-hedrite with $i-2$ central circuits (see Theorem [6), which is, moreover, pure.

Conjecture 1 An irreducible $i$-hedrite is maximal irreducible if and only if it has $i-2$ central circuits.

\section{Classification of pure irreducible $i$-hedrites}

The easiest case, $i=4$, of i-hedrites admits following complete characterization:

Theorem 5 (i) Any 4-hedrite can be obtained from some 4-hedrite with two central circuits by simultaneous $t_{1}$ - and $t_{2}$-inflation along those circuits; it is irreducible if and only if $t_{1}=t_{2}=1$.

(ii) Any 4-hedrite with two central circuits is defined by its number of vertices $n$ and by shift $j, 0 \leq j \leq n / 4$ with $\operatorname{gcd}(n / 2, j)=1$, vertices between the pair of boundary 2-gons on the horizontal circuit (see, for example, 4-hedrite 8-1) and the remaining pair of 2-gons. Remark that several different values of shift can yield the same graph.

(iii) Any 4-hedrite is balanced.

Proof. (i) and (ii) are proved in DeSt02, while (iii) is obvious for 4-hedrite with two central circuits and remain true under $t_{1}$ - and $t_{2}$-inflation.

The shift $j=0$ corresponds to $\mathbf{2 - 1}$ and its only-on-one-circuit $m$-inflations $J_{4, n=2 m}$. The shift $j=1$ corresponds to $\mathbf{4 - 1}$ and $I_{4, n=2 m+2}$. Denote by $K_{4,4 m}$, for any $m \geq 2$, any $4 m$-vertex 4-hedrite obtained from 4 -1 by $m$-inflation of only one its central circuit; so, its CC-vector is $\left(4^{m}, 4 m\right)$, its symmetry is $D_{2 d}$ and it is reducible. Clearly, any $K_{4, n=4 m}$ has the maximal shift $j=n / 4$.

Theorem 6 Any pure irreducible $i$-hedrite is, either any 4-hedrite with two central circuits, or a 5-hedrite 6-2, or one of 6-hedrites 8-6, 14-20, or one of the following eight 8-hedrites: 6-1, 12-4, 12-5, 14-7, and (see Figure馬) 20-1, 22-1, 30-1, 32-1.

Proof. Let $G$ be a pure irreducible $i$-hedrite having $r$ central circuits. If one deletes a central circuit, then, in general, 1-gon can appear. It does not happen for $G$, since it would imply a self-intersection of a central circuit. So, the result of deletion of a central circuit from $G$ produces an pure irreducible $i$-hedrite with $r-1$ central circuits. 


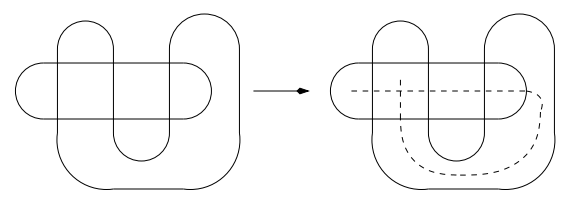

Figure 4: No pure irreducible $i$-hedrite can be obtained by cutting of the above 4-hedrite

First, if $r=2$, then the Theorem 5 from DeSt02 gives that such $G$ are exactly 4-hedrites with two central circuits; all of them are classified in Theorem 5 .

We prove the Theorem by systematic analysis of all possible ways to add to $G$ (for $r=2,3,4,5$ ) a central circuit, in order to get a pure irreducible $i$-hedrite with $r+1$ central circuits.

Let $r=2$. Then $G$ can be only one of two smallest 4-hedrites. In fact, if $G$ is another 4-hedrite, then, because of classification Theorem [5] it has a form as in Figure 4 .

New central circuit should cut both 2-gons on opposite edges, since, otherwise, there is a rail-road. But Figure 4]shows, on example for $n=6$, that a self-intersection appears if two central circuits intersect in more than four vertices.

So, the only possible 4-hedrites with two central circuits, which can be cut in order to produce irreducible pure $\boldsymbol{i}$-hedrite are 4 -hedrites $\mathbf{2 - 1}$ and $\mathbf{4 - 1}$. All cases are indicated below.

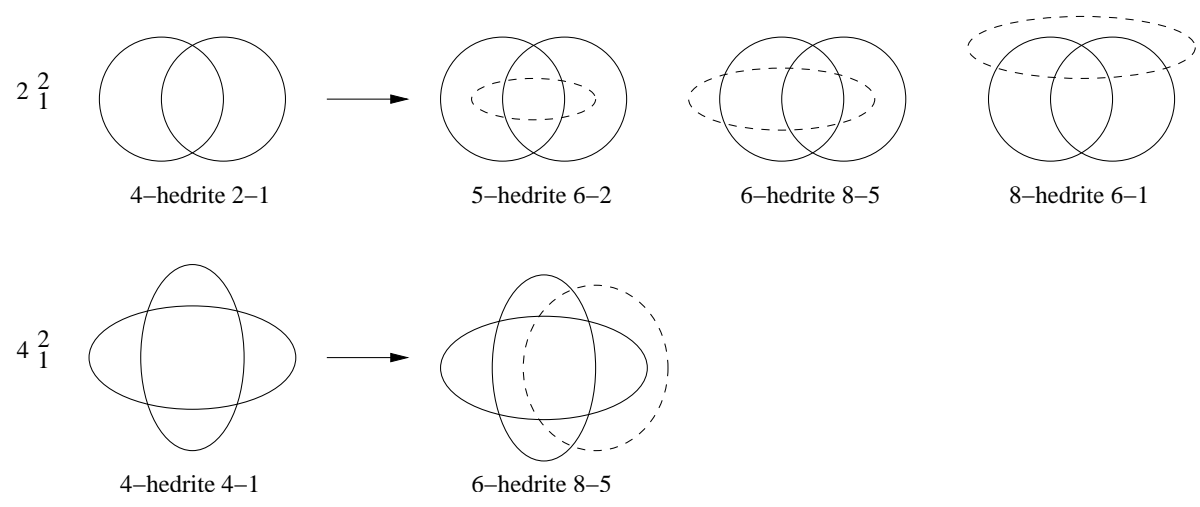

Now, all irreducible pure $i$-hedrites with three central circuits are 5 -hedrite $\mathbf{6 - 2}$, 6-hedrite 8-5 and 8-hedrite 6-1 (i.e. the projections of links $6_{1}^{3}, 8_{6}^{3}$ and $6_{2}^{3}$ ). In fact, we apply the same procedure to those three $i$-hedrites; see picture below: 


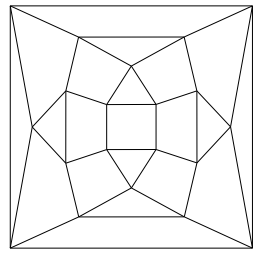

Nr.20-1 $D_{2 d}$

$\left(8^{5}\right)$

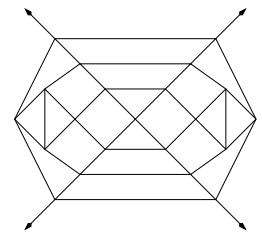

Nr.22-1 $D_{2 h}$

$\left(8^{3}, 10^{2}\right)$

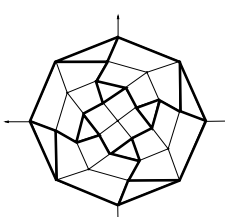

Nr.30-1 $O$

$\left(10^{6}\right)$

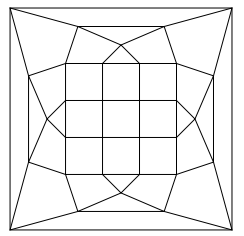

Nr.32-1 $D_{4 h}$ $\left(10^{4}, 12^{2}\right)$

Figure 5: Pure irreducible $i$-hedrites with 5 or 6 central circuits

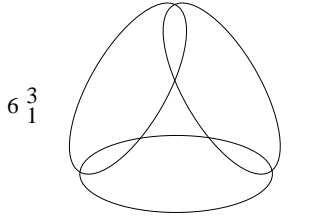

5-hedrite 6-2

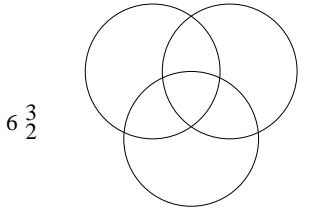

8-hedrite 6-1

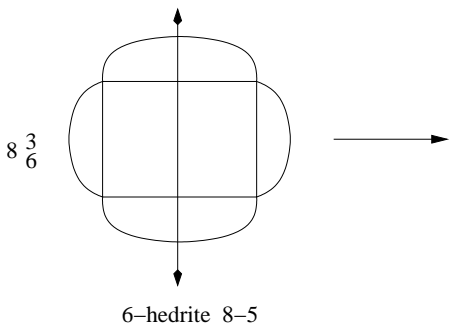

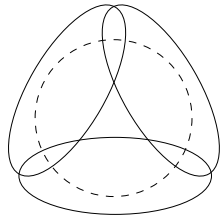

8-hedrite $12-5$

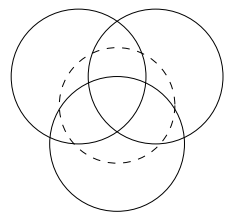

8-hedrite 12-4

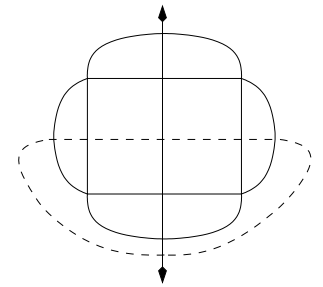

8-hedrite 14-7

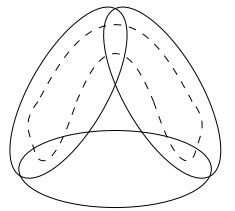

6-hedrite 14-20

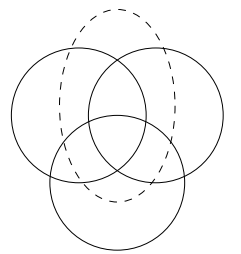

8-hedrite 12-5

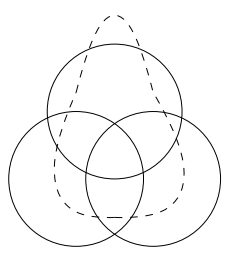

8-hedrite 14-7

Next, all irreducible pure $i$-hedrites with four central circuits are 8-hedrites 12-4, 12-5, 14-7 and 6-hedrite 14-20.

By the same method, one can see that there are exactly two pure irreducible $i$-hedrites with five central circuits and two with six central circuits (see Figure 5 ).

Remark 3 Any pure $i$-hedrite comes from a pure irreducible $i$-hedrite with, say, $j$ central circuits by simultaneous $t_{1}-, \ldots, t_{j}$-inflation along those circuits; it is irreducible if and only if $t_{1}=\cdots=t_{j}=1$.

\section{$8 \quad$ Symmetry groups of $i$-hedrites}

We consider below the maximal symmetry groups of plane graphs; these groups are identified with the corresponding point groups. 
Theorem 7 We indicate here the lists of symmetry groups of $i$-hedrites, together with the smallest number of vertices, for which they appear:

(i) The only symmetry groups of 4-hedrites are point subgroups of $D_{4 h}$, which contain $D_{2}$ as point subgroup, i.e. $D_{4 h}(n=2), D_{4}(n=10), D_{2 h}(n=4)$, $D_{2 d}(n=6), D_{2}(n=12)$.

(ii) The only symmetry groups of 5-hedrites are: $C_{1}(n=10), C_{2}(n=8), C_{s}(n=$ 7), $C_{2 v}(n=5), D_{3}(n=15), D_{3 h}(n=3)$.

(iii) The only symmetry groups of 6-hedrites are: $D_{2 d}(n=4), D_{2 h}(n=6)$ and all their point subgroups, i.e. $D_{2}(n=12), C_{2 h}(n=10), C_{2 v}(n=5), C_{i}(16 \leq n \leq$ 30), $C_{2}(n=6), C_{s}(n=9), C_{1}(n=9)$.

(iv) The only symmetry groups of 7-hedrites are point subgroups of $C_{2 v}$, i.e. $C_{2 v}(n=$ $7), C_{2}(n=11), C_{s}(n=8), C_{1}(n=11)$.

(v) The only symmetry groups of 8-hedrites are: $C_{1}(n=16), C_{s}(n=14), C_{2}(n=$ 12), $C_{2 v}(n=11), C_{i}(22 \leq n \leq 46), C_{2 h}(22 \leq n \leq 26), S_{4}(22 \leq n \leq 60)$, $D_{2}(n=10), D_{2 d}(n=14), D_{2 h}(n=22), D_{3}(n=18), D_{3 d}(n=12), D_{3 h}(n=$ 9), $D_{4}(n=18), D_{4 d}(n=8), D_{4 h}(n=10), O(n=30), O_{h}(n=6)$.

Proof. For 4-hedrites, see DeSt02. Any transformation stabilizing a 2-gon, can interchange its two edges and two vertices. So, the stabilizing point subgroup of a 2-gon can be $C_{2 v}, C_{s}, C_{2}$ or $C_{1}$ only.

The unique 2-gon of a 7 -hedrite has to be preserved by the symmetry group; so, all possibilities are: $C_{2 v}, C_{s}, C_{2}, C_{1}$.

Every symmetry of an $i$-hedrite induces a symmetry on its 2 -gons and 3 -gons. Since the stabilizer of a 2-gon, 3-gon has maximal size 4, 6, this imply that the order of the symmetry group of an $i$-hedrite is bounded from above by $4|\operatorname{Sym}(8-i)|=$ $4(8-i) !$ and $6|\operatorname{Sym}(2 i-8)|=6(2 i-8)$ !.

So, in particular, the order of symmetry group of an 6 -hedrite is at most 8 . If $f$ is an element of order three, then it fixes each of two 2-gons. Since the stabilizer of a 2 -gon does not contain an element of order three, no such $f$ exists. If $f$ is a rotation of order 4 , then $f^{2}$ is a rotation of order 2 stabilizing each 2 -gon; so, the axis of $f$ goes through the two 2-gons. This is a contradiction. By a search in the Tables of the groups one can see that the only possibilities are: $C_{1}, C_{s}, C_{2}, C_{i}, C_{2 v}, C_{2 h}, D_{2}$, $D_{2 h}, D_{2 d}$. But there exists a 6 -hedrite for any of such symmetries, in Figure 6 and subsection 9.3 below.

For 5-hedrites, since there are two 3-gons, the maximal order of the group is 12 . The oddness of the number of 2-gons excludes central symmetry, axis of order 4 , and groups $D_{2}, D_{2 h}, D_{2 d}$. If $G$ is a 5 -hedrite with a 3 -fold axis then this axis goes through the two 3-gons, say, $T_{1}$ and $T_{2}$. If one consider a belt of 4 -gons around $T_{1}$, then, after a number $p$ of steps, one will encounter a 2-gon and so, by symmetry, three 2 -gons. So, we will have the following possibilities for $p=1$ : 

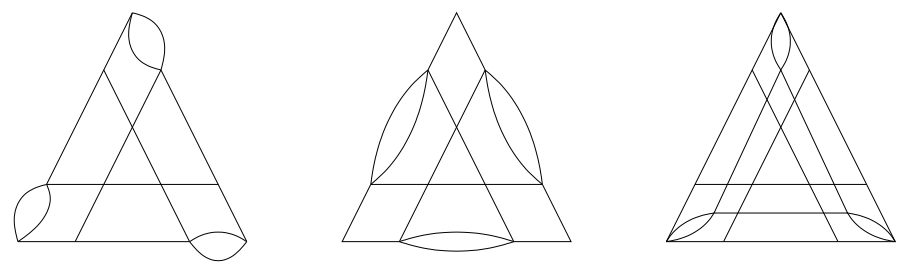

There is only one way to extend this graph to a 5 -hedrite and the obtained extension has symmetry at least $D_{3}$. So, the group is $D_{3}, D_{3 h}$ or $D_{3 d}$.

An 8-hedrite $G$ has $k$-fold axis of rotation with $k=2,3$ or 4 . If $k=3$, then the axis of the rotation goes through two 3 -gons, say $T_{1}$ and $T_{8}$. If one consider, around 3 -gon $T_{1}$, a belt of 4 -gons, then, after some number $p$ of steps, one will encounter a 3 -gon and so, by symmetry, three 3 -gons, say, $\left\{T_{2}, T_{3}, T_{4}\right\}$. Adding, if necessary, belts of 4 -gons, one will encounter the last three 3 -gons, say, $\left\{T_{5}, T_{6}, T_{7}\right\}$. The patch formed by the six triangles $T_{2}, \ldots, T_{7}$ has symmetry $D_{3}$ at least and so, $G$ has also this symmetry. Consequently, the symmetry of $G$ is $D_{3}, D_{3 h}$ or $D_{3 d}$.

If $k=4$, then the axis of the rotation goes through a vertex or a 4-gon. Assume, for simplicity, that this axis goes through a vertex; then, by repeating above reasoning, one obtains two orbits of 3-gons, say, $\left\{T_{1}, T_{2}, T_{3}, T_{4}\right\}$ and $\left\{T_{5}, T_{6}, T_{7}, T_{8}\right\}$, under 4 -fold symmetry and the symmetry group is $D_{4}, D_{4 h}, D_{4 d}, O$ or $O_{h}$.

So, one obtains the above list of 18 possible point groups. All these groups appear in subsection 9.5 (groups $O_{h}, D_{4 d}, D_{3 h}, D_{2}, D_{4 h}, C_{2 v}, D_{3 d}, C_{2}$ ), in Figure 6 (groups $D_{2 d}, C_{s}, C_{1}, D_{4}, D_{3}, C_{2 h}, C_{i}, S_{4}$ ) and in Figure 5 (groups $D_{2 h}, O$ ); for all groups (except, possibly, $C_{i}, C_{2 h}$ and $S_{4}$ ) those examples of 8-hedrites have smallest number of vertices.

Remark 4 The simplest case, $i=4$, of $i$-hedrites admits following characterization for each of its five possible groups. It has symmetry $D_{2 h}$ (respectively, $D_{2 d}$ ) if and only if it is an $t$-inflation, for some $t \geq 1, m \geq 2$, of $J_{4,2 m}$ or $I_{4,2 m+2}$ with even $m$ (respectively, of $K_{4,4 m}$ or $I_{4,2 m+2}$ with odd $m$ ). Any 4-hedrite with group $D_{4}$ or $D_{4 h}$ has $2\left(k^{2}+l^{2}\right)$ vertices for some $k \geq l \geq 0$ (the group $D_{4 h}$ corresponds to the case $l=k$ or 0$)$; it comes from the smallest 4-hedrite 2-1 by Goldberg-Coxeter construction (see Gold37], Cox71] and [DD03]). All other 4-hedrites have symmetry $D_{2}$; in shift terms, they are exactly those, for which interchange of central circuits changes the value of shift.

We expect, that a 7-hedrite with the highest symmetry $C_{2 v}$ exists for any $n \geq 10$.

\section{$9 \quad$ Small $i$-hedrites}

Here and below all links are given in Rolfsen's notation (see the table in Rol76] and also, for example, Kaw96) for links with at most 9 crossings and knots with 10 crossings, or, otherwise, in Dowker-Thistlewhaite's numbering (see [Thi]), if any. We write $\sim$ if the projection in the pictures and Table below is different from the one given in corresponding cases above. 


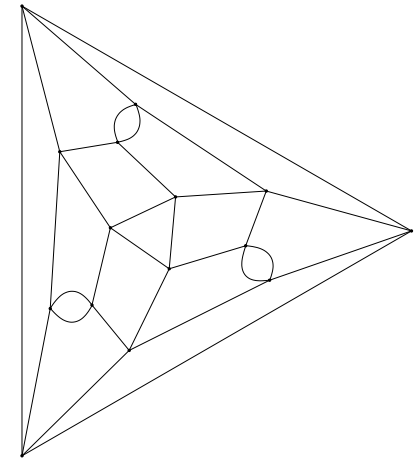

5-hedrite 15-1 $D_{3}$

smallest (30)

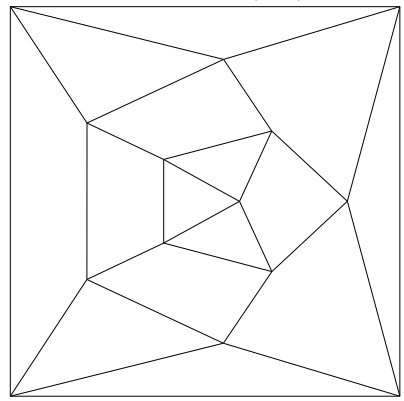

8-hedrite 14-2 $C_{s}$ smallest $(6 ; 22)$

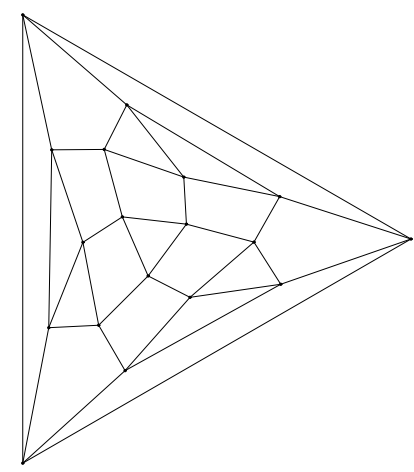

8-hedrite 18-2 $D_{3}$

smallest (36)

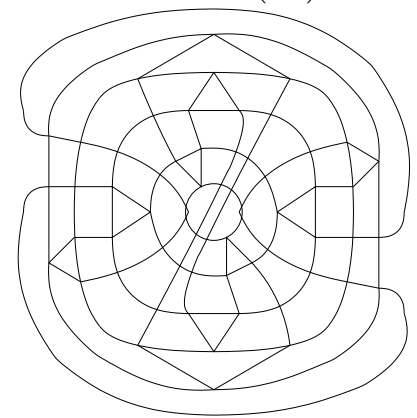

8-hedrite 60-1 $S_{4}$

$\left(16 ; 26^{4}\right)$

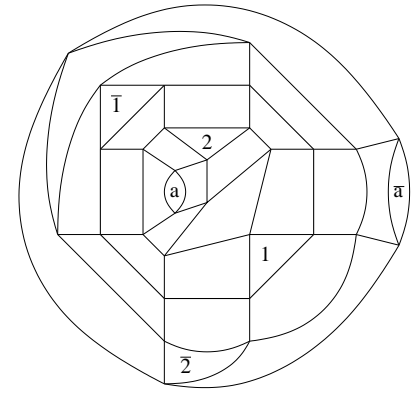

6-hedrite 30-1 $C_{i}$ $\left(8 ; 26^{2}\right)$

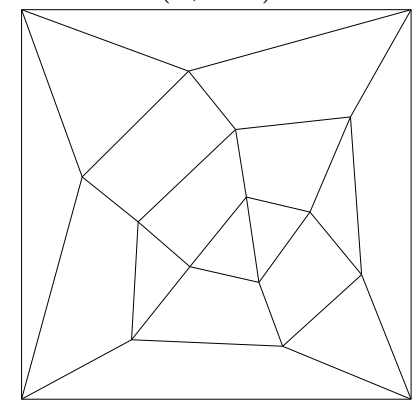

8-hedrite 16-1 $C_{1}$ smallest $(6,8 ; 18)$

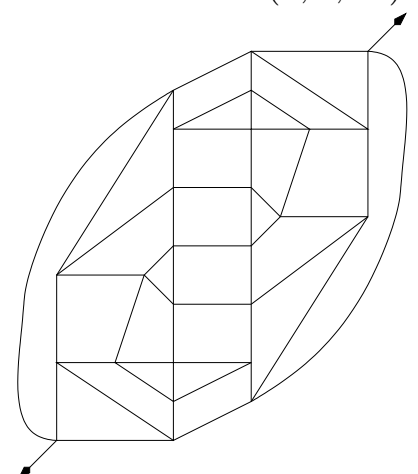

8-hedrite 26-1 $C_{2 h}$ $\left(8^{2} ; 36\right)$

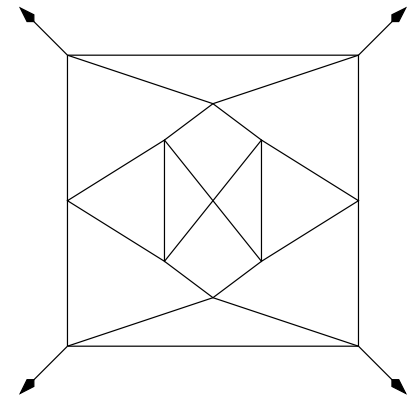

8-hedrite 14-4 $D_{2 d}$ smallest $\left(14^{2}\right)$

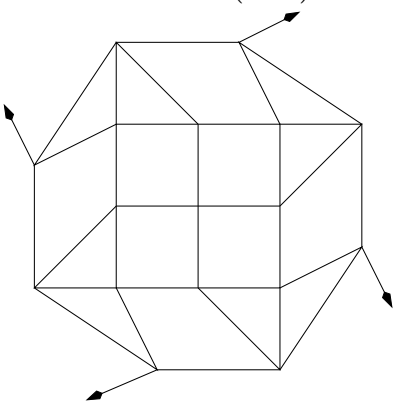

8-hedrite 18-1 $D_{4}$ smallest $\left(18^{2}\right)$

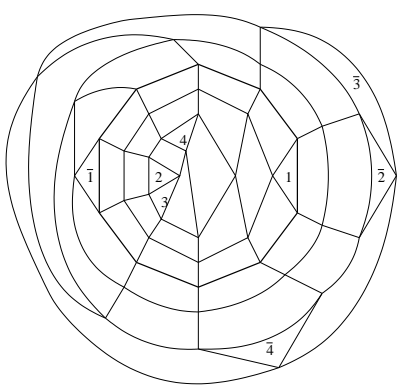

8-hedrite 46-1 $C_{i}$

$(10 ; 82)$

Figure 6: Some $i$-hedrites with special symmetry groups 
We give on the pictures below all $i$-hedrites with at most 12 vertices, indicating under picture of each its symmetry, CC-vector and corresponding alternating link. If an $i$-hedrite is 2 -connected but not 3 -connected, then we add a symbol $*$ just after the number. If an $i$-hedrite is reducible, i.e. has a rail-road, then we add mention "red.". All $i$-hedrites with 13, 14 and 15 vertices are listed in Table 2,

Only three reducible $i$-hedrites with $n \leq 15$ have self-intersecting railroad: 5 hedrites 12-3, 14-6 and 6-hedrite 13-11.

On the pictures below, in order to express better the (maximal) symmetry of an $i$-hedrite, we put:

(i) a double arrow, in order to represent an edge passing at infinity,

(ii) a quadruple arrow, in order to represent a vertex at infinity.

\subsection{4-hedrites}

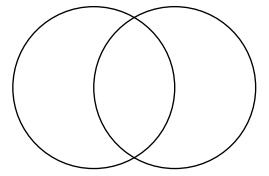

Nr.2-1 $D_{4 h}$

$2_{1}^{2} \quad\left(2^{2}\right)$

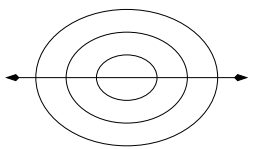

Nr.6-2* $D_{2 h}$ $3 \times 2_{1}^{2} \quad\left(2^{3}, 6\right)$ red.

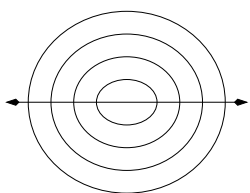

Nr.8-4* $D_{2 h}$ $4 \times 2_{1}^{2} \quad\left(2^{4}, 8\right)$ red.

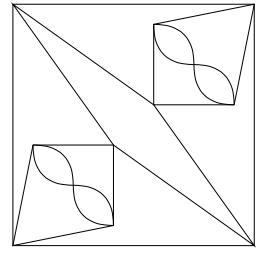

Nr.12-1 ${ }^{*} \quad D_{2 h}$

???? $\left(12^{2}\right)$

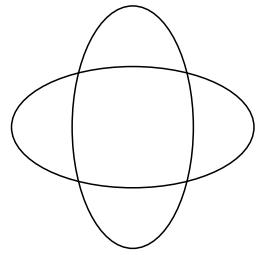

Nr.4-1 $D_{4 h}$ $4_{1}^{2} \quad\left(4^{2}\right)$

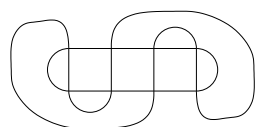

Nr.8-1* ${ }^{*} \quad D_{2 h}$ $\sim 8_{4}^{2} \quad\left(8^{2}\right)$

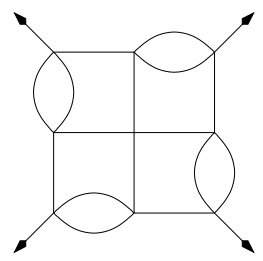

Nr.10-1 $D_{4}$ $10_{121}^{2} \quad\left(10^{2}\right)$

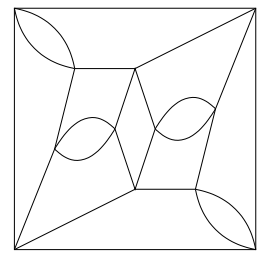

Nr.12-2 $D_{2}$ ???? $\left(6^{2}, 12\right)$ red.

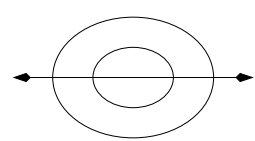

Nr.4-2 ${ }^{*} \quad D_{2 h}$ $2 \times 2_{1}^{2} \quad\left(2^{2}, 4\right)$ red.

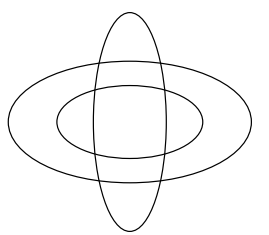

Nr.8-2 $D_{2 d}$

$8_{4}^{3} \quad\left(4^{2}, 8\right)$ red.

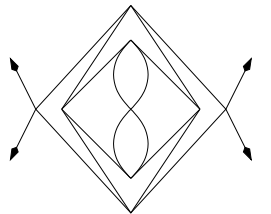

Nr.10-2* $D_{2 d}$

$\sim 10_{120}^{2} \quad\left(10^{2}\right)$

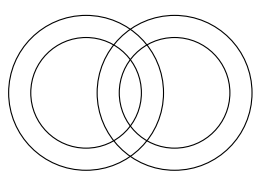

Nr.8-3 $D_{4 h}$

$8_{1}^{4} \quad\left(4^{4}\right)$ red.

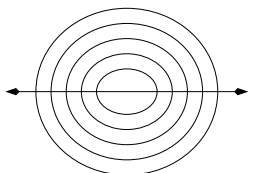

Nr.10-3* $D_{2 h}$ $5 \times 2_{1}^{2} \quad\left(2^{5}, 10\right)$ red.

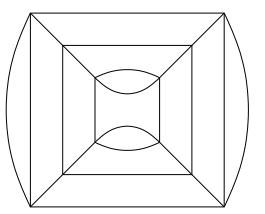

Nr.12-3 $D_{2 d}$ ???? $\left(4^{3}, 12\right)$ red.

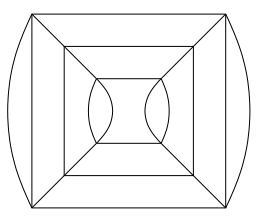

Nr.12-4 $D_{2 h}$ ???? $\left(4^{3}, 6^{2}\right)$ red. 


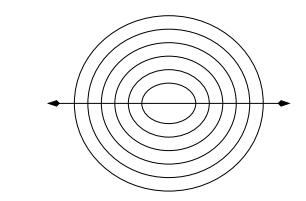

Nr.12-5* ${ }^{*} \quad D_{2 h}$

$6 \times 2_{1}^{2} \quad\left(2^{6}, 12\right)$ red.

\section{$9.2 \quad 5$-hedrites}

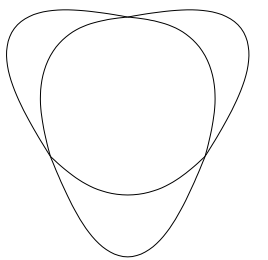

Nr.3-1 $D_{3 h}$

$3_{1} \quad$ (6)

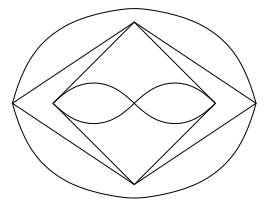

Nr.7-1 ${ }^{*} \quad C_{2 v}$

$\sim 7_{5} \quad(14)$

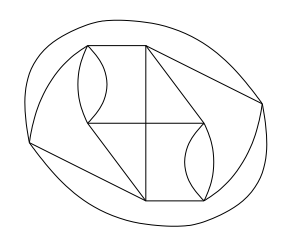

Nr.9-1 $C_{2}$

$9_{38} \quad(18)$

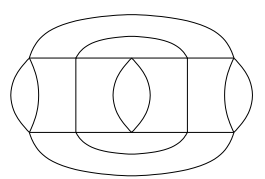

Nr.10-3 $C_{2 v}$

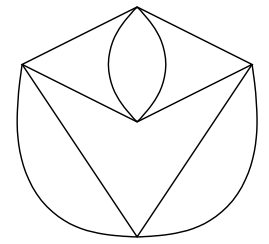

Nr.5-1* $C_{2 v}$

$5_{2} \quad(10)$
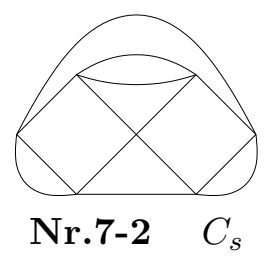

$7_{5}^{2} \quad(4 ; 10)$

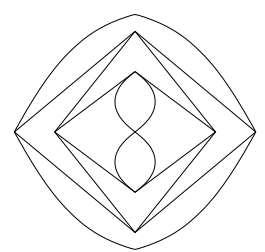

Nr.9-2* $C_{2 v}$

$\sim 9_{18} \quad(18)$

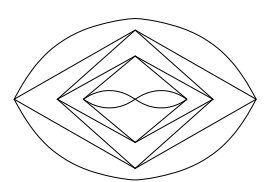

Nr.11-1* $C_{2 v}$

$\sim 11_{236}$

(22)

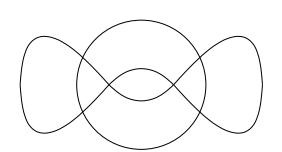

Nr.6-1 $C_{2 v}$

$6_{3}^{2} \quad(4 ; 8)$

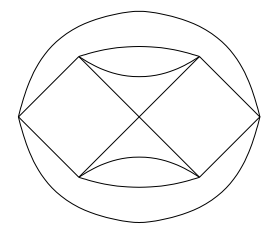

Nr.7-3 $C_{2 v}$

$7_{1}^{3} \quad\left(4^{2} ; 6\right)$

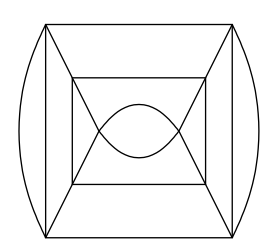

Nr.10-1 $C_{2 v}$

$10_{155}^{3} \quad\left(4^{2} ; 12\right)$ red.

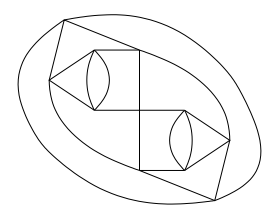

Nr.11-2 $C_{2}$

$\sim 11_{124} \quad(22)$

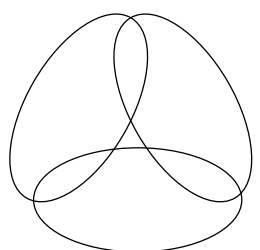

Nr.6-2 $D_{3 h}$ $6_{1}^{3} \quad\left(4^{3}\right)$

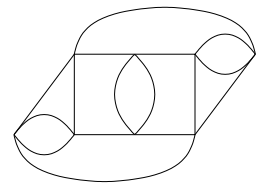

Nr.8-1 $C_{2}$ $\sim 8_{15} \quad(16)$

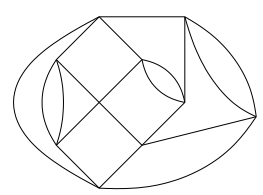

Nr.10-2 $C_{1}$ $10_{85}^{2} \quad(6 ; 14)$

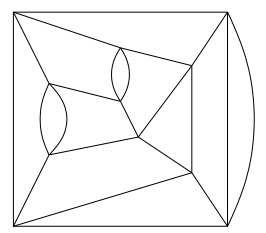

Nr.11-3 $C_{1}$ $11_{226}^{2} \quad(6 ; 16)$ 


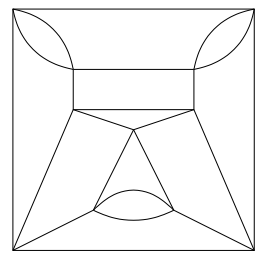

Nr.11-4 $C_{s}$

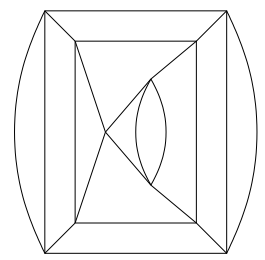

Nr.11-5 $C_{s}$

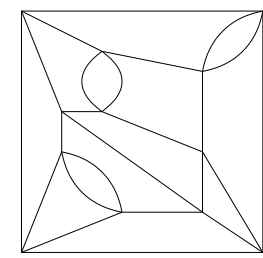

Nr.12-1 $C_{1}$

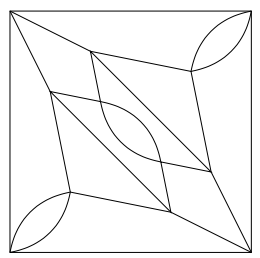

Nr.12-2 $C_{2 v}$ ???? $\left(12^{2}\right)$

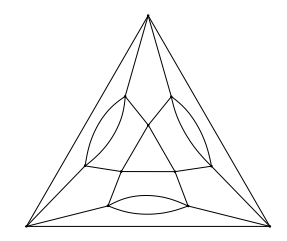

Nr.12-3 $D_{3 h}$

???? $\left(12^{2}\right)$ red.

\subsection{6-hedrites}

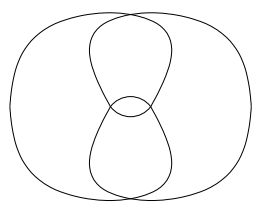

Nr.4-1 $D_{2 d}$

$4_{1} \quad$ (8)

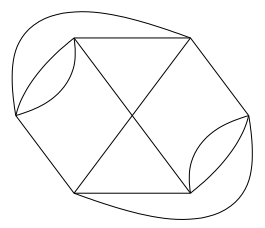

Nr.7-1 $C_{2}$

$\sim 7_{7} \quad$ (14)

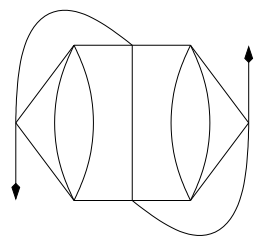

Nr.8-4 $C_{2}$

$\sim 8_{8}^{2} \quad(6 ; 10)$

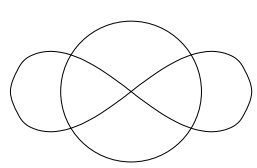

Nr.5-1 $C_{2 v}$

$5_{1}^{2} \quad(4 ; 6)$

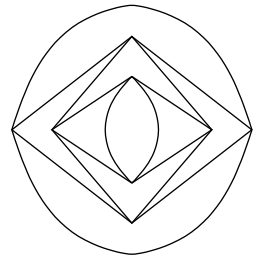

Nr.8-1* $D_{2 d}$
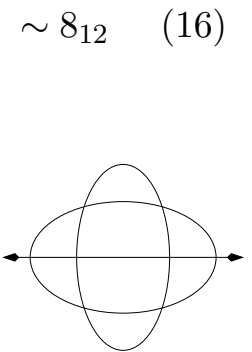

Nr.8-5 $D_{2 h}$

$8_{6}^{3} \quad\left(4,6^{2}\right)$

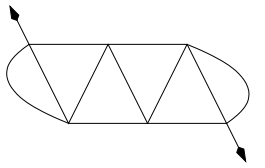

Nr.6-1 $C_{2}$

$6_{3} \quad(12)$

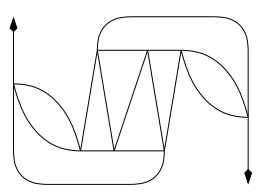

Nr.8-2 $C_{2}$

$8_{17} \quad$ (16)

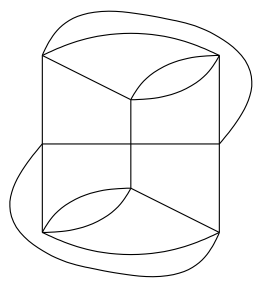

Nr.9-1 $C_{2}$

$\sim 9_{31} \quad$ (18)

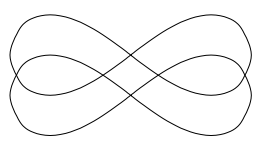

Nr.6-2* $D_{2 h}$

$\sim 6_{3}^{2} \quad\left(6^{2}\right)$

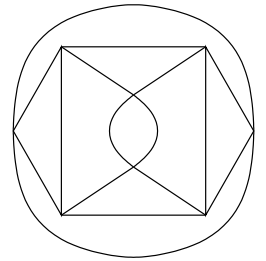

Nr.8-3 $D_{2 d}$

$8_{14}^{2} \quad(4 ; 12)$

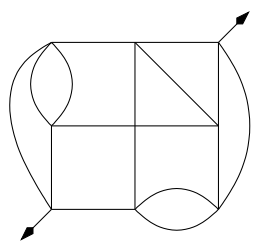

Nr.9-2 $C_{1}$

$9_{33} \quad$ (18) 


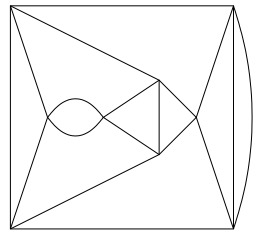

Nr.9-3 $C_{s}$ $9_{38}^{2} \quad(4 ; 14)$

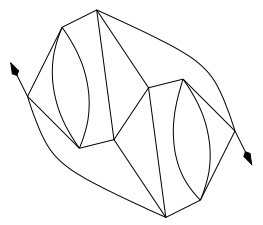

Nr.10-2 $C_{2}$

$\sim 10_{88} \quad(20)$

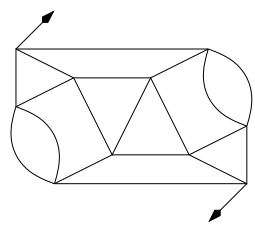

Nr.10-6 $C_{2}$ $10_{86}^{2} \quad(6 ; 14)$

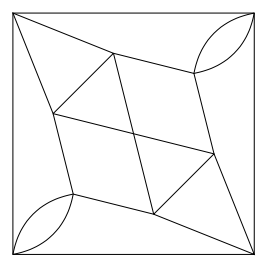

Nr.11-1 $C_{2 v}$

$11_{332} \quad$ (22)

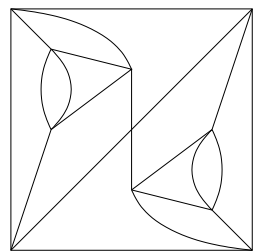

Nr.11-5 $C_{2}$ ???? $(8 ; 14)$

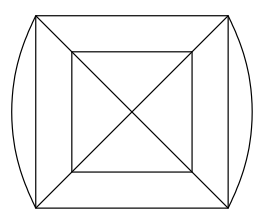

Nr.9-4 $C_{2 v}$ $9_{12}^{3} \quad\left(4^{2} ; 10\right)$ red.

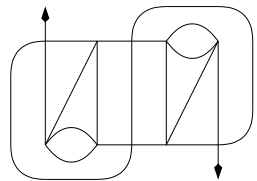

Nr.10-3 $C_{2}$

$\sim 10_{45} \quad(20)$

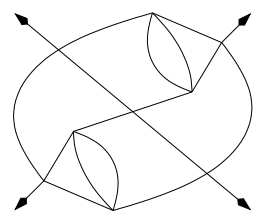

Nr.10-7 $C_{2}$ $10_{43}^{2} \quad(4 ; 16)$

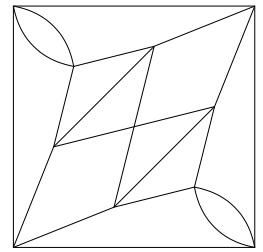

Nr.11-2 $C_{2 v}$ $11_{297} \quad$ (22)

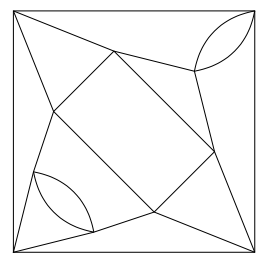

Nr.11-6 $C_{s}$ ???? $(8,14)$

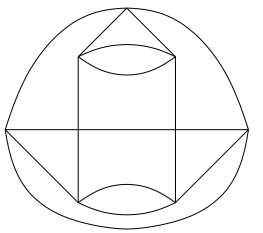

Nr.9-5 $C_{s}$

$9_{11}^{3} \quad(4,6 ; 8)$

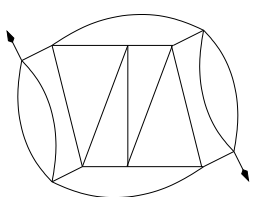

Nr.10-4 $C_{2}$

$10_{115} \quad$ (20)

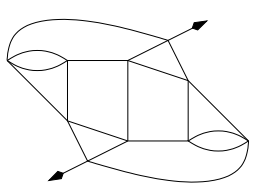

Nr.10-8 $C_{2 h}$

$\sim 10_{136}^{3} \quad\left(4 ; 8^{2}\right)$

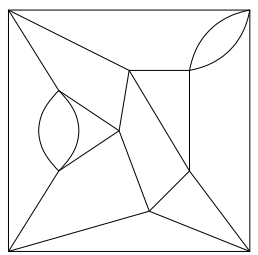

Nr.11-3 $C_{1}$

$\sim 11_{125}$

(22)

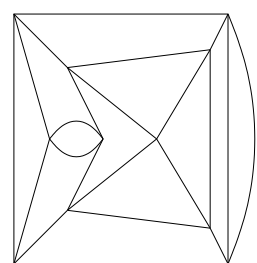

Nr.11-7 $C_{s}$

$11_{351}^{2} \quad(10,12)$

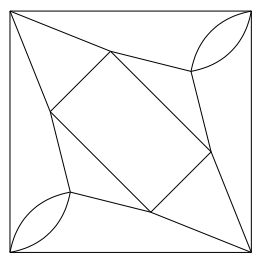

Nr.10-1 $C_{2 v}$ $10_{120} \quad$ (20)

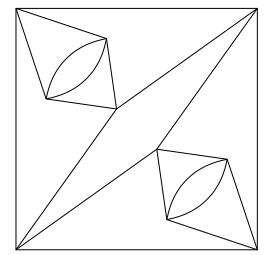

Nr.10-5* ${ }^{*} \quad D_{2 h}$ $\sim 10_{87}^{2} \quad\left(10^{2}\right)$

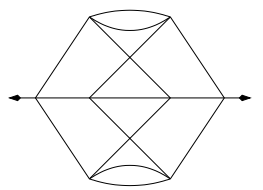

Nr.10-9 $C_{2 v}$ $10_{136}^{3} \quad(4,6 ; 10)$

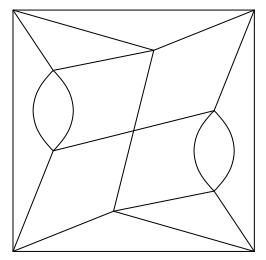

Nr.11-4 $C_{2}$ $11_{317}^{2} \quad(8 ; 14)$

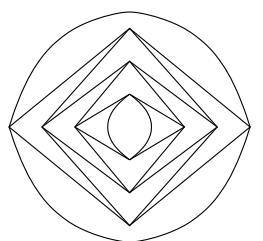

Nr.12-1* ${ }^{*} \quad D_{2 d}$ $\sim 12_{477} \quad$ (24) 


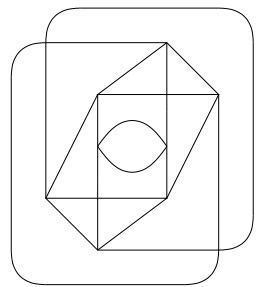

Nr.12-2 $D_{2}$

$12_{1152} \quad$ (24)

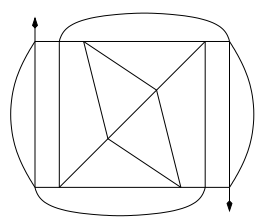

Nr.12-6 $C_{2}$

$12_{1167} \quad$ (24)

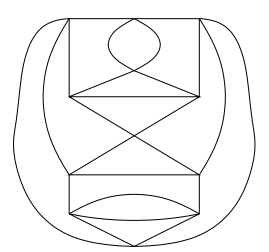

Nr.12-10 $C_{s}$ ???? $(8,16)$

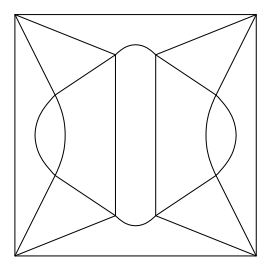

Nr.12-14 $D_{2 h}$ ???? $\left(4^{2}, 8^{2}\right)$ red.
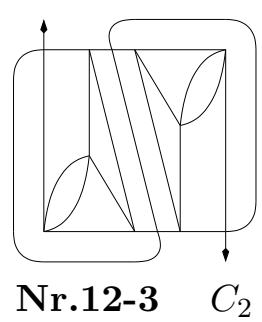

$\sim 12_{499} \quad$ (24)

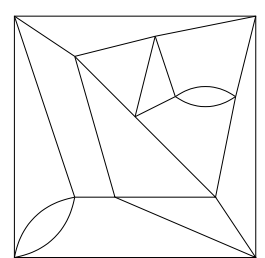

Nr.12-7 $C_{1}$

$\sim 12_{626}$

(24)

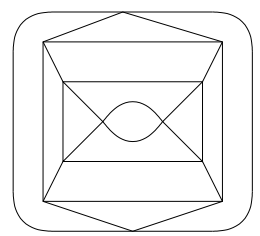

Nr.12-11 $D_{2 d}$ ???? $\left(4^{2} ; 16\right)$ red.

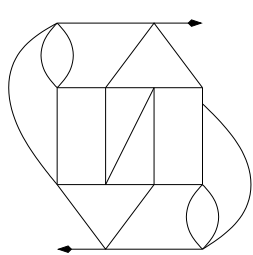

Nr.12-4 $C_{2}$

$\sim 12_{458} \quad$ (24)

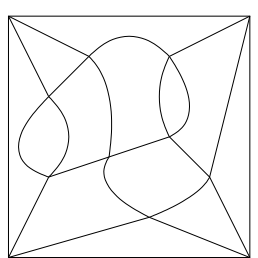

Nr.12-8 $C_{1}$

???? $(6 ; 18)$

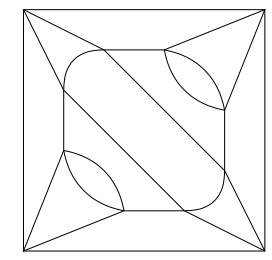

Nr.12-12 $C_{2 v}$

???? $\left(8 ; 8^{2}\right)$

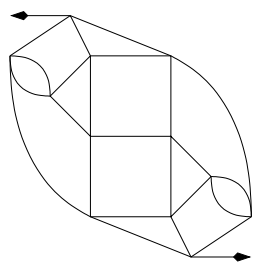

Nr.12-5 $C_{2}$

$12_{1102} \quad$ (24)

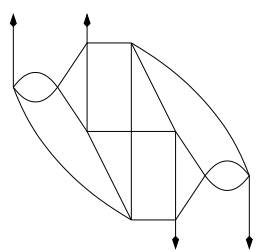

Nr.12-9 $C_{2}$

???? $(10,14)$

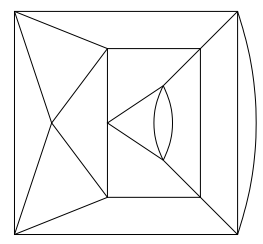

Nr.12-13 $C_{s}$ ???? $(6 ; 8,10)$

\subsection{7-hedrites}

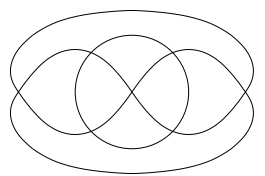

Nr.7-1 $C_{2 v}$

$7_{6}^{2} \quad(4 ; 10)$

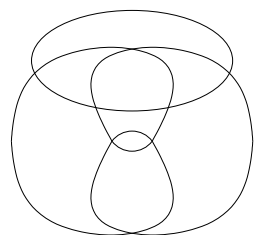

Nr.8-1 $C_{s}$

$8_{13}^{2} \quad(4 ; 12)$

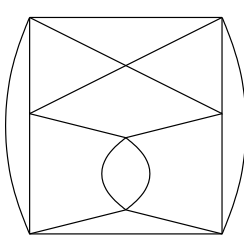

Nr.9-1 $C_{s}$

$9_{34} \quad$ (18)

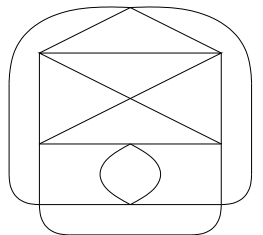

Nr.10-1 $C_{s}$ $10_{121} \quad$ (20) 


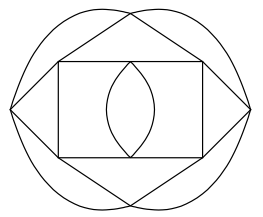

Nr.10-2 $C_{2 v}$ $10_{111}^{2} \quad\left(10^{2}\right)$

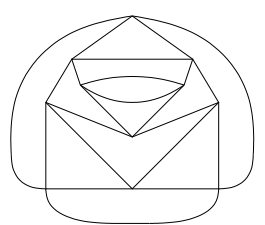

Nr.11-3 $C_{s}$ $11_{150}^{2} \quad(8,14)$

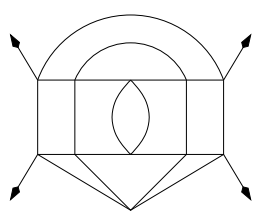

Nr.12-3 $C_{s}$ ???? $(10,14)$

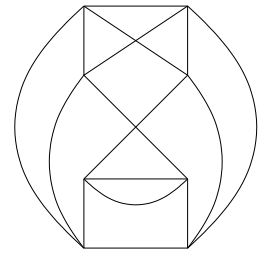

Nr.10-3 $C_{s}$ $\sim 10_{69}^{2} \quad(8,12)$

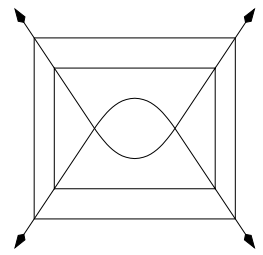

Nr.11-4 $C_{2 v}$ $11_{487}^{3} \quad\left(4^{2} ; 14\right)$ red.

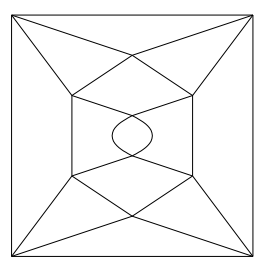

Nr.12-4 $C_{2 v}$ ???? $\left(6^{2} ; 12\right)$

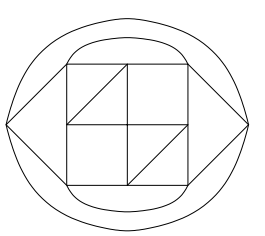

Nr.11-1 $C_{2}$

$11_{288} \quad$ (22)

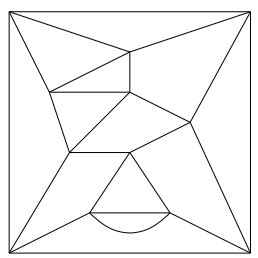

Nr.12-1 $C_{1}$

$\sim 12_{361}$

(24)

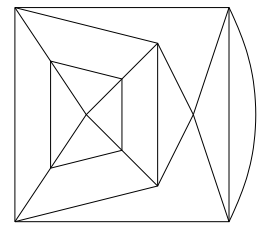

Nr.12-5 $C_{s}$ ???? $\left(4^{2} ; 16\right)$ red.

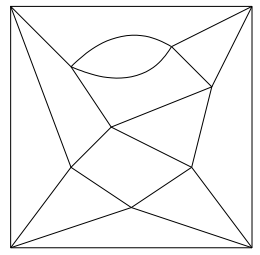

Nr.11-2 $C_{1}$

$11_{301}$ (22)

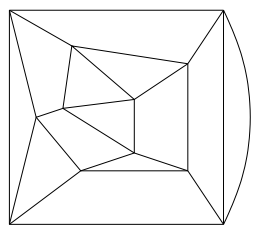

Nr.12-2 $C_{1}$ ???? $(6 ; 18)$

\subsection{8-hedrites}

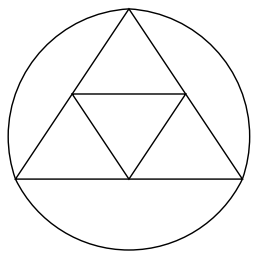

Nr.6-1 $O_{h}$

$6_{2}^{3} \quad\left(4^{3}\right)$

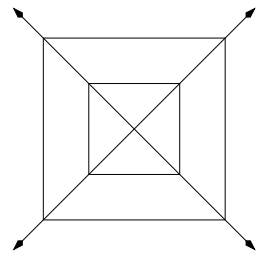

Nr.10-2 $D_{4 h}$

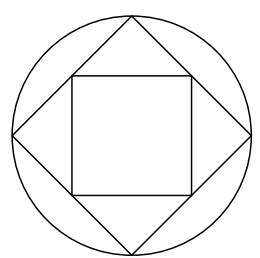

Nr.8-1 $D_{4 d}$

$8_{18} \quad$ (16)

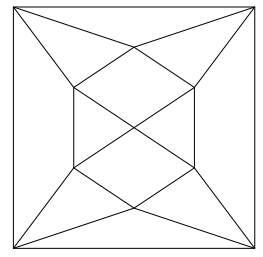

Nr.11-1 $C_{2 v}$ $11_{520}^{3} \quad\left(6^{2} ; 10\right)$

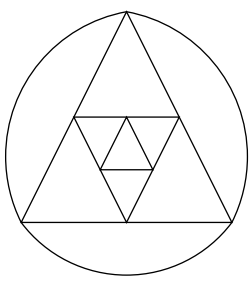

Nr.9-1 $D_{3 h}$

$9_{40} \quad$ (18)

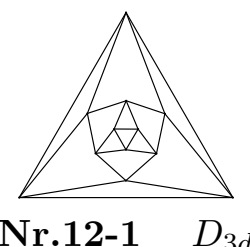

$12_{1019} \quad$ (24)

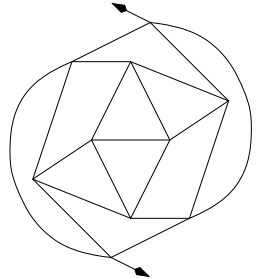

Nr.10-1 $D_{2}$ $10_{56}^{2} \quad(6 ; 14)$

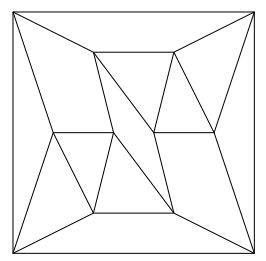

Nr.12-2 $D_{2}$

$12_{868} \quad$ (24) 


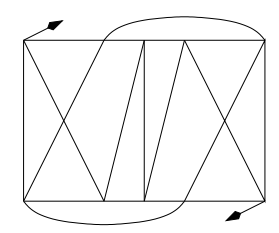

Nr.12-3 $C_{2}$

???? (6;18)

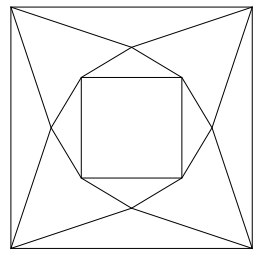

Nr.12-4 $O_{h}$

????

$\left(6^{4}\right)$

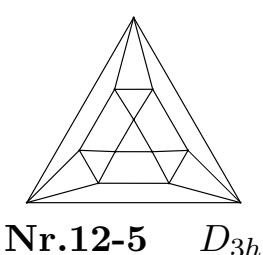

$\left(6^{4}\right)$ 


\begin{tabular}{|c|c|c|c|}
\hline Nr. & Group & CC-vector & alt.knot \\
\hline \multicolumn{4}{|c|}{ 4-hedrites } \\
\hline $14-1^{*}$ & $\overline{D_{2 d}}$ & $14^{2}$ & \\
\hline $14-2$ & $D_{2}$ & $14^{2}$ & \\
\hline $14-3^{*}$ & $D_{2 h}$ & $2^{7}, 14$ red. & \\
\hline \multicolumn{4}{|c|}{ 5-hedrites } \\
\hline $13-1^{*}$ & $C_{2 v}$ & 26 & 133097 \\
\hline 13-2 & $C_{1}$ & 26 & 134054 \\
\hline 13-3 & $C_{2}$ & $6^{2} ; 14$ & \\
\hline $13-4$ & $C_{s}$ & $6 ; 8,12$ & \\
\hline 14-1 & $\overline{C s}$ & 28 & $14_{16368}$ \\
\hline 14-2 & $C_{1}$ & $6 ; 22$ & \\
\hline 14-3 & $C_{1}$ & $8 ; 20$ & \\
\hline 14-4 & $C_{2 v}$ & $8^{2} ; 12$ & \\
\hline 14-5 & $C_{2 v}$ & $4^{3} ; 16$ red. & \\
\hline $14-6$ & $C_{2 v}$ & $6^{2} ; 8^{2}$ red. & \\
\hline $14-7$ & $C_{2 v}$ & $4^{3}, 8^{2}$ red. & \\
\hline 15-1 & $D_{3}$ & 30 & 1583814 \\
\hline 15-2 & $C_{2 v}$ & 30 & $15_{54593}$ \\
\hline 15-3 & $C_{s}$ & 30 & 1583824 \\
\hline $15-4$ & $C_{2}$ & 30 & $15_{20161}$ \\
\hline $15-5$ & $C_{s}$ & 12,18 & \\
\hline 15-6 & $C_{s}$ & 14,16 & \\
\hline $15-7$ & $C_{1}$ & $8 ; 22$ & \\
\hline $15-8$ & $C_{2 v}$ & $8^{2} ; 14$ & \\
\hline $15-9$ & $C_{s}$ & $4^{3} ; 18$ red. & \\
\hline 15-10 & $C_{s}$ & $4^{3}, 8 ; 10$ red. & \\
\hline \multicolumn{4}{|c|}{ 6-hedrites } \\
\hline 13-1 & $C_{2}$ & 26 & $\sim 13_{1739}$ \\
\hline 13-2 & $C_{2}$ & 26 & 133586 \\
\hline 13-3 & $C_{2}$ & 26 & $\sim 13_{1345}$ \\
\hline $13-4$ & $C_{s}$ & 26 & $13_{3811}$ \\
\hline 13-5 & $C_{1}$ & 26 & $13_{1485}$ \\
\hline 13-6 & $C_{1}$ & 26 & 133957 \\
\hline $13-7$ & $C_{1}$ & 26 & $\sim 13_{2957}$ \\
\hline $13-8$ & $C_{1}$ & $8 ; 18$ & \\
\hline 13-9 & $C_{s}$ & 12,14 & \\
\hline 13-10 & $C_{s}$ & $4^{2} ; 18$ red. & \\
\hline 13-11 & $C_{2 v}$ & $8^{2}, 10$ red. & \\
\hline 13-12 & $C_{2 v}$ & $8^{2} ; 10$ & \\
\hline 13-13 & $C_{2 v}$ & $4^{3} ; 14$ red. & \\
\hline 13-14 & $C_{s}$ & $4^{2}, 8 ; 10$ red. & \\
\hline 14-1 & $C_{2 h}$ & 28 & $14_{17173}$ \\
\hline 14-2 & $C_{2}$ & 28 & $14_{17079}$ \\
\hline 14-3 & $C_{2}$ & 28 & 148767 \\
\hline $14-4$ & $C_{2}$ & 28 & $14_{17734}$ \\
\hline 14-5 & $C_{2}$ & 28 & $14_{17148}$ \\
\hline $14-6$ & $C_{1}$ & 28 & $14_{17309}$ \\
\hline $14-7$ & $C_{1}$ & 28 & $14_{5570}$ \\
\hline $14-8$ & $C_{2}$ & $6 ; 22$ & \\
\hline 14-9 & $C_{2}$ & $6 ; 22$ & \\
\hline 14-10 & $C_{2}$ & $10 ; 18$ & \\
\hline 14-11 & $C_{2}$ & $10 ; 18$ & \\
\hline 14-12 & $C_{s}$ & 10,18 & \\
\hline $14-13^{*}$ & $D_{2 h}$ & $14^{2}$ & \\
\hline $14-14$ & $C_{2 v}$ & $14^{2}$ & \\
\hline 14-15 & $\mathrm{C}_{2}$ & 12,16 & \\
\hline $14-16$ & $C_{s}$ & 12,16 & \\
\hline 14-17 & $C_{2}$ & $4^{2} ; 20$ red. & \\
\hline 14-18 & $C_{2}$ & $8 ; 10^{2}$ & \\
\hline 14-19 & $C_{2}$ & $6^{2} ; 16$ & \\
\hline $14-20$ & $D_{2 h}$ & $6^{2}, 8^{2}$ & \\
\hline 14-21 & $C_{2 v}$ & $6^{3}, 10$ red. & \\
\hline
\end{tabular}

\begin{tabular}{|c|c|c|c|}
\hline $\begin{array}{l}14-22 \\
14-23\end{array}$ & $\begin{array}{l}C_{2 h} \\
C_{2 v}\end{array}$ & $\begin{array}{l}4^{2} ; 10^{2} \text { red. } \\
4^{2}, 8 ; 12 \text { red. }\end{array}$ & \\
\hline 15-1 & $C_{2}$ & 30 & $\sim 15_{39533}$ \\
\hline $15-2$ & $C_{2}$ & 30 & $15_{66949}$ \\
\hline $15-3$ & $C_{2}$ & 30 & 1583008 \\
\hline $15-4$ & $C_{1}$ & 30 & 1545248 \\
\hline $15-5$ & $C_{1}$ & 30 & $\sim 15_{20975}$ \\
\hline $15-6$ & $C_{1}$ & 30 & $15_{64488}$ \\
\hline $15-7$ & $C_{1}$ & 30 & $\sim 15_{45357}$ \\
\hline $15-8$ & $C_{1}$ & $6 ; 24$ & \\
\hline $15-9$ & $C_{1}$ & $6 ; 24$ & \\
\hline 15-10 & $C_{2 v}$ & $8 ; 22$ & \\
\hline 15-11 & $C_{1}$ & $8 ; 22$ & \\
\hline 15-12 & $C_{1}$ & $8 ; 22$ & \\
\hline 15-13 & $C_{s}$ & 10,20 & \\
\hline $15-14$ & $C_{1}$ & 10,20 & \\
\hline 15-15 & $C_{2 v}$ & $8 ; 10,12$ & \\
\hline 15-16 & $C_{2 v}$ & $6^{2}, 8 ; 10$ & \\
\hline $15-17$ & $C_{s}$ & $6^{3} ; 12$ red. & \\
\hline \multicolumn{4}{|c|}{ 7-hedrites } \\
\hline 13-1 & $C_{s}$ & 26 & 133861 \\
\hline $13-2$ & $C_{1}$ & 26 & $13_{3769}$ \\
\hline 13-3 & $C_{1}$ & $6 ; 20$ & \\
\hline $13-4$ & $C_{1}$ & 10,16 & \\
\hline $13-5$ & $C_{s}$ & 10,16 & \\
\hline $13-6$ & $C_{2 v}$ & $6^{2} ; 14$ & \\
\hline $13-7$ & $C_{s}$ & $6^{2} ; 14$ & \\
\hline 14-1 & $C_{1}$ & 28 & $14_{13725}$ \\
\hline $14-2$ & $C_{1}$ & 28 & $14_{10841}$ \\
\hline $14-3$ & $C_{1}$ & 28 & $14_{5714}$ \\
\hline $14-4$ & $C_{1}$ & 28 & $14_{14207}$ \\
\hline $14-5$ & $C_{1}$ & $6 ; 22$ & \\
\hline $14-6$ & $C_{s}$ & 10,18 & \\
\hline $14-7$ & $C_{2}$ & $14^{2}$ & \\
\hline $14-8$ & $C_{s}$ & $6^{2} ; 16$ & \\
\hline 14-9 & $C_{2 v}$ & $6^{2} ; 16$ & \\
\hline 15-1 & $C_{2}$ & 30 & 1582225 \\
\hline $15-2$ & $C_{1}$ & 30 & $15_{60207}$ \\
\hline $15-3$ & $C_{1}$ & 30 & 1580242 \\
\hline $15-4$ & $C_{s}$ & $6 ; 24$ & \\
\hline $15-5$ & $C_{1}$ & $6 ; 24$ & \\
\hline $15-6$ & $C_{1}$ & $6 ; 24$ & \\
\hline $15-7$ & $C_{1}$ & 10,20 & \\
\hline $15-8$ & $C_{1}$ & 10,20 & \\
\hline 15-9 & $C_{2 v}$ & 14,16 & \\
\hline $15-10$ & $C_{1}$ & 14,16 & \\
\hline 15-11 & $C_{2 v}$ & $6^{2} ; 18$ & \\
\hline 15-12 & $C_{2 v}$ & $4^{3} ; 18$ red. & \\
\hline \multicolumn{4}{|c|}{ 8-hedrites } \\
\hline $13-1$ & $C_{2}$ & 26 & $13_{3478}$ \\
\hline 13-2 & $C_{2 v}$ & $6^{2} ; 14$ & \\
\hline 14-1 & $C_{2}$ & 28 & $14_{17895}$ \\
\hline 14-2 & $C_{s}$ & $6 ; 22$ & \\
\hline $14-3$ & $D_{2}$ & $6 ; 22$ & \\
\hline $14-4$ & $D_{2 d}$ & $14^{2}$ & \\
\hline 14-5 & $C_{2}$ & $6^{2} ; 16$ & \\
\hline $14-6$ & $D_{2}$ & $8 ; 10^{2}$ & \\
\hline 14-7 & $D_{4 h}$ & $6^{2}, 8^{2}$ & \\
\hline $14-8$ & $D_{4 h}$ & $4^{3}, 8^{2}$ red. & \\
\hline $15-1$ & $C_{2}$ & 30 & 1582477 \\
\hline $15-2$ & $C_{s}$ & $6 ; 24$ & \\
\hline $15-3$ & $C_{s}$ & $6 ; 24$ & \\
\hline $15-4$ & $C_{2}$ & $8 ; 22$ & \\
\hline $15-5$ & $D_{3 h}$ & $10^{3}$ & \\
\hline
\end{tabular}

Table 2: All $i$-hedrites with 13, 14 and 15 vertices 


\section{References}

[Cox71] H.S.M.Coxeter, Virus macromolecules and geodesic domes, in A spectrum of mathematics; ed. by J.C.Butcher, Oxford University Press/Auckland University Press: Oxford, U.K./Auckland New-Zealand, (1971) 98-107.

[DDF02] M.Deza, M.Dutour and P.W.Fowler, Zigzags, Rail-roads and Knots in Fullerenes, submitted.

[DeGr99] M.Deza and V.P.Grishukhin, $l_{1}$-embeddable polyhedra, in: Algebras and Combinatorics, Int. Congress CAC '97 Hong Kong, ed. by K.P. Shum et al., Springer-Verlag (1999) 189-210.

[DHL02] M.Deza, T.Huang and K-W.Lih, Central Circuit Coverings of Octahedrites and Medial Polyhedra, Journal of Math. Research \& Exposition 22-1 (2002) 4966 .

[DeSt02] M.Deza and M.Shtogrin, Octahedrites, Symmetry, Special Issue "Polyhedra and Science and Art", 2002.

[Dut] M.Dutour, PlanGraph, a gap package for Planar Graph, in preparation.

[DD02] M.Dutour, M.Deza, Zigzag Structure of Simple Bifaced Polyhedra, submitted.

[DD03] M.Dutour, M.Deza, Goldberg-Coxeter Construction for convex polyhedra, in preparation.

[GaKe94] M.L.Gargano and J.W.Kennedy, Gaussian graphs and digraphs, Congressus Numerantium 101 (1994) 161-170.

[GoRo01] C.Godsil and G.Royle, Algebraic Graph Theory, Graduate Texts in Mathematics 207, Springer-Verlag, Berlin - New York, 2001.

[Gold37] M.Goldberg, A class of multisymmetric polyhedra, Tohoku Math. Journal, 43 (1937) 104-108.

[Grün67] B.Grünbaum, Convex polytopes, Interscience, New York, 1967.

[Grün72] B.Grünbaum, Arrangements and Spreads, Regional Conference Series in Mathematics 10, American Mathematical Society, 1972.

[GrünMo63] B.Grünbaum and T.S.Motzkin, The number of hexagons and the simplicity of geodesics on certain polyhedra, Canadian Journal of Mathematics 15 (1963) 744-751.

[Harb97] H.Harborth, Eulerian straight ahead cycles in drawings of complete bipartite graphs, Bericht 97/23, Institute für Mathematik, Tech. Universität Braunschweg, 1997. 
[Heid98] O.Heidemeier, Die Erzeugung von 4-regulären, planaren, simplen, zusammenhängenden Graphen mit vorgegebenen Flächentypen, Diplomarbeit, Universität Bielefeld, Fakultät für Wirtschaft und Mathematik, 1998.

[Jeo95] D.Jeong, Realizations with a cut-through Eulerian circuit, Discrete Mathematics 137 (1995) 265-275.

[Kaw96] A.Kawauchi, A survey of knot theory, Birkhäuser, 1996.

[Kir85] T. Kirkman, The enumeration, description, and construction of knots with fewer than 10 crossings, Trans. Roy. Soc. Edin. 32 (1885), 281-309.

[Kot69] A.Kotzig, Eulerian lines in finite 4-valent graphs and their transformations, in: Theory of Graphs, Proceedings of a colloquium, Tihany 1966, ed. by P.Erdos and G.Katona, Academic Press, New York (1969) 219-230.

[Liu98] Liu Yanpei, Embedding in Graphs, Kluwer, Dodrecht, 1998.

[PTZ96] T.Pisanski, T.Tucker and A.Zitnik, Eulerian Embedding of Graphs, University of Ljubljana, IMMF Preprint Series 34 (1996) 531.

[Rol76] D.Rolfsen, Knots and Links, Mathematics Lecture Series 7, Publish or Perish, Berkeley, 1976; second corrected printing: Publish or Perish, Houston, 1990 .

[Sha75] H.Shank, The Theory of Left-Right Paths, in: Combinatorial Mathematics III, Proceedings of 3rd Australian Conference, St Lucia 1974, Lecture Notes in Mathematics 452, Springer-Verlag, Berlin - New York (1975), pp. 42-54.

[Thi] M.Thistlewaite, Homepage, http://www.math.utk.edu/〜morwen. 


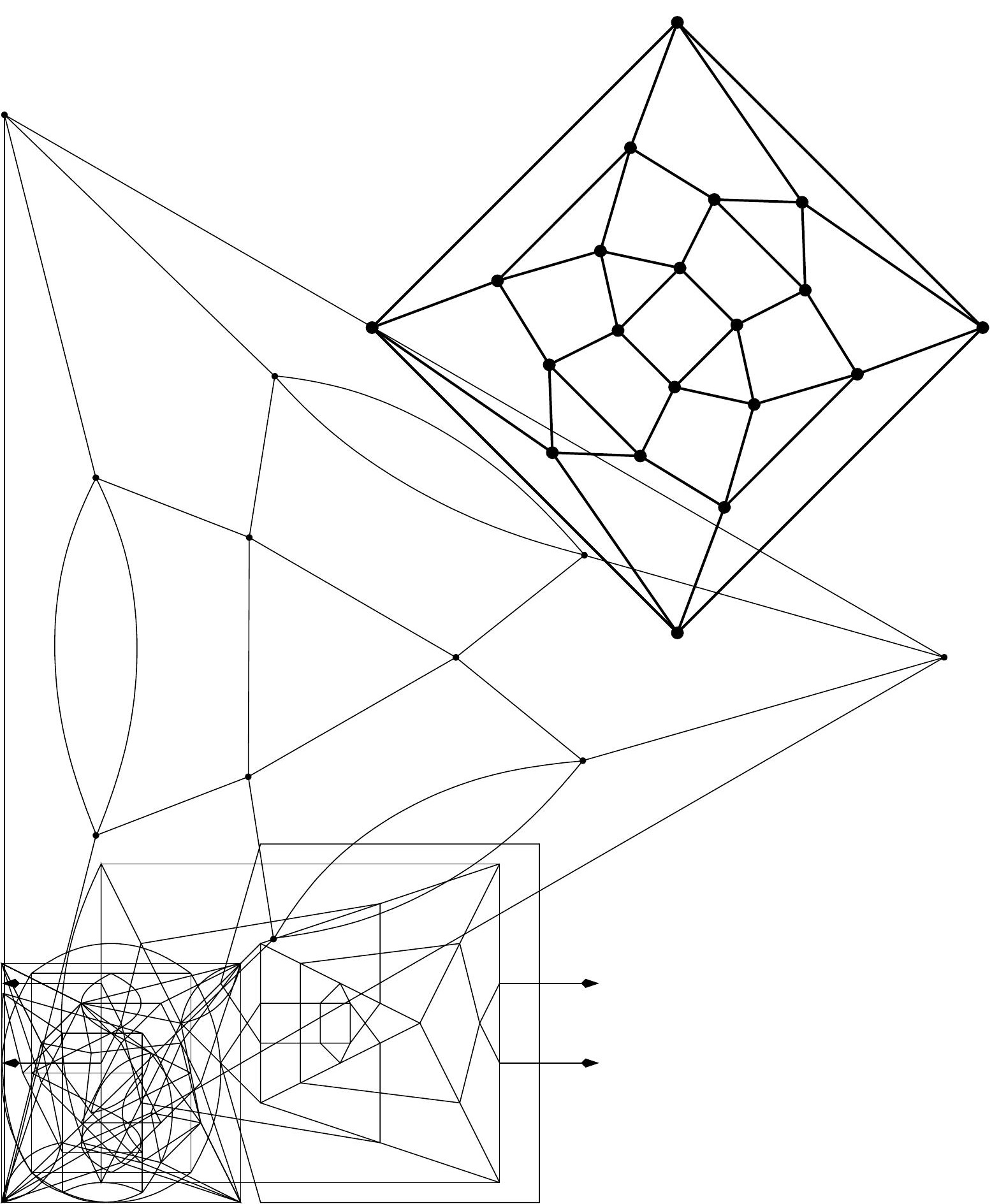

